\title{
En Route to D-Ring Inverted Phorbol Esters
}

Sharon Chow, Tanja Krainz, Paul V. Bernhardt, and Craig M. Williams*

School of Chemistry and Molecular Biosciences, University of Queensland, Brisbane, 4072, Australia

Supporting Information

\section{Table of Contents}

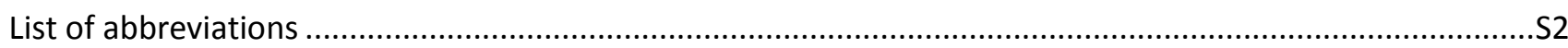

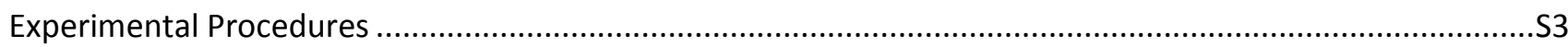

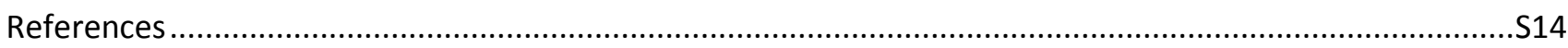

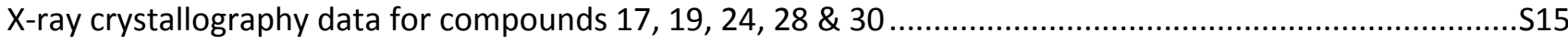

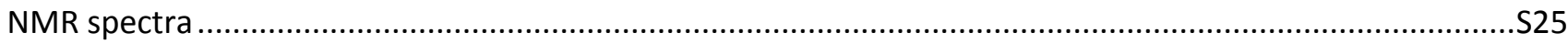




\section{List of abbreviations}

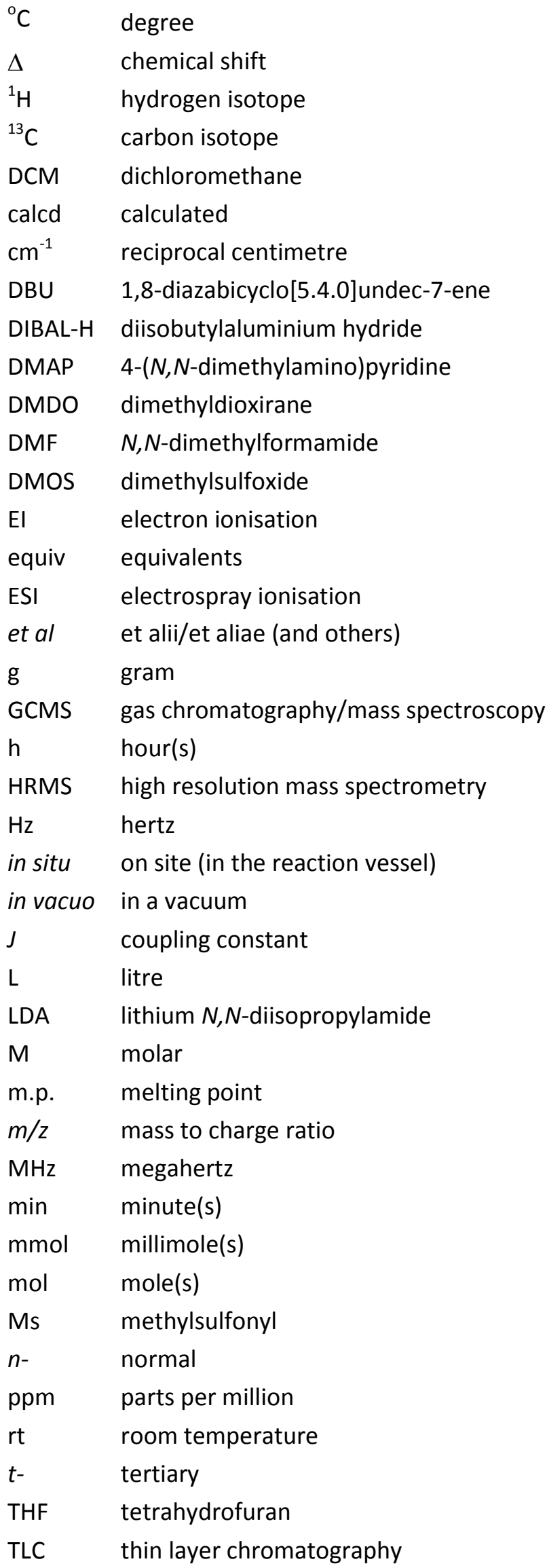




\section{Experimental Procedures}

\section{General information}

${ }^{1} \mathrm{H}$ and ${ }^{13} \mathrm{C}$ NMR spectra were recorded on Bruker AV300 (300.13 MHz; $\left.75.47 \mathrm{MHz}\right)$, AV400 (400.13 MHz; 100.62 MHz), AV500/AS500 (500.13 MHz; $125.76 \mathrm{MHz}$ ) or AS700 (700.47 MHz; $176.13 \mathrm{MHz}$ ) instruments. Coupling constants are given in hertz $(\mathrm{Hz})$ and chemical shifts are reported as $\delta$ values in parts-per-million $(\mathrm{ppm})$ with the solvent resonance as the internal standard $\left({ }^{1} \mathrm{H} \mathrm{NMR}_{-} \mathrm{CDCl}_{3}: \delta 7.26\right.$ and ${ }^{13} \mathrm{C} \mathrm{NMR}-\mathrm{CDCl}_{3}: \delta$ 77.0, ${ }^{1} \mathrm{H}$ NMR- $\mathrm{C}_{6} \mathrm{D}_{6}: \delta 7.16$ and ${ }^{13} \mathrm{C}$ NMR- $\left.\mathrm{C}_{6} \mathrm{D}_{6}: \delta 128.39\right)$. Data are reported as followed: chemical shift, multiplicity ( $\mathrm{s}=$ singlet, $\mathrm{br} \mathrm{s}=$ broad singlet, $\mathrm{d}=$ doublet, $\mathrm{t}=$ triplet, $\mathrm{q}=$ quartet, quin = quintuplet, $\mathrm{sext}=$ sextuplet, spt = septet, $m=$ multiplet), coupling constants $(\mathrm{Hz})$, and integration. $\mathrm{HRMS}$ measurements were recorded in positive ionisation mode on a Bruker MicrOTOF-Q instrument with a Bruker ESI source using electron ionisation. Sodium formate was used as a reference calibrant. Column chromatography was performed using distilled solvents on silica (silica gel $230-400$ mesh) or aluminium oxide (neutral). Anhydrous DCM, triethylamine, and cyclohexane were distilled from calcium hydride under argon before use. Anhydrous THF was freshly distilled from sodium under argon before use. All catalysts used in this work [TPAP, $\mathrm{Rh}_{2}(\mathrm{OAC})_{4}$ and $\mathrm{AuCl}$ ] were sourced from Sigma. Infrared spectroscopy (IR) was acquired using a Perkin Elmer-400 model, with the scanning range of 4000 to $650 \mathrm{~cm}^{-1}$. Melting points are uncorrected. XRay crystallographic data, obtained from the University of Queensland, was carried out on an Oxford Diffraction Gemini S Ultra CCD diffractometer using Cu-K $\alpha$ radiation ( $=1.54180 \AA$ ). The crystals were kept at $190 \mathrm{~K}$ using an Oxford Cryosystems Desktop Cooler. 
<smiles>COC1=CC(=O)C=C(C)C1=O</smiles>

\section{2-Methoxy-6-methylphenol (15)}

According to the literature procedure, ${ }^{1} \mathrm{o}$-vanillin (12) $(19.7 \mathrm{~g}, 0.123 \mathrm{~mol})$, hydrazine hydrate $(55-60 \%$ wt. in water, $25 \mathrm{~mL}$, approx. $0.280 \mathrm{~mol})$ and triethylene glycol $(100 \mathrm{~mL})$ were mixed and then heated to $110{ }^{\circ} \mathrm{C}$. Potassium hydroxide $(45.5 \mathrm{~g}, 0.813 \mathrm{~mol}$ ) was then added in portions to the bright yellow solution over 20 30 min. Effervesce was observed after each addition of the base. The resulting orange solution was stirred at $150{ }^{\circ} \mathrm{C}$ for $20 \mathrm{~h}$. After cooling to room temperature, the reaction was carefully poured into ice cold hydrochloric acid $(\mathrm{HCl})(1 \mathrm{M}, 450 \mathrm{~mL})$ and further acidified with additional concentrated $\mathrm{HCl}$ until reaching $\mathrm{pH}$ 2. A white precipitate was formed during this process. The mixture was then extracted with chloroform $\left(4 \times 80 \mathrm{~mL}\right.$ ). The combined extracts were washed with brine, dried over anhydrous $\mathrm{MgSO}_{4}$ and then concentrated under reduced pressure to give a pale orange liquid that still contained a small amount of chloroform. The crude phenol 13 was separated into 2 portions for the oxidation process. The first portion of the crude phenol $(9.36 \mathrm{~g}, 67.7 \mathrm{mmol})$ was taken up in DMF (60 mL) and added salcomine $14(2.14 \mathrm{~g}, 6.08$ $\mathrm{mmol}$ ). Oxygen was then gently bubbled into the brown suspension for $1 \mathrm{~d}$, or until no starting material was detected by TLC. A mixture of ethyl acetate and diethyl ether $(1: 1,300 \mathrm{~mL})$ was then added and stirred for $30 \mathrm{~min}$. The suspension was then filtered via a pad of celite and the pad was rinsed with fresh solvent $(100 \mathrm{~mL})$. The combined filtrates were washed with water $(3 \times 200 \mathrm{~mL})$. The aqueous layers were then reextracted with fresh solvent $(3 \times 100 \mathrm{~mL})$. On combining all the organic extracts, washes were performed firstly with a saturated $\mathrm{NaHCO}_{3}$ solution $(50 \mathrm{~mL})$ and then brine $(80 \mathrm{~mL})$ respectively. Upon drying with $\mathrm{MgSO}_{4}$ and concentrating under reduced pressure, the residue was purified by flash silica gel chromatography (DCM, collected in $100 \mathrm{~mL}$ fractions) to give the titled product 15 as a yellow solid. The total product weight was measured at $17.3 \mathrm{~g}$ (88\% over 2 steps) after repeating the oxidation with the second half of the crude phenol. Spectroscopic data and the melting point matched that described in literature. ${ }^{2,3}$

m.p. $148-149^{\circ} \mathrm{C}$

${ }^{1} \mathrm{H}$ NMR $\left(300 \mathrm{MHz}, \mathrm{CDCl}_{3}\right) \delta 2.07(\mathrm{~d}, \mathrm{~J}=1.5 \mathrm{~Hz}, 3 \mathrm{H}), 3.82(\mathrm{~s}, 3 \mathrm{H}), 5.88(\mathrm{~d}, \mathrm{~J}=2.5 \mathrm{~Hz}, 1 \mathrm{H}), 6.53-6.55(\mathrm{~m}, 1 \mathrm{H})$.

${ }^{13} \mathrm{CNMR}\left(100 \mathrm{MHz}, \mathrm{CDCl}_{3}\right) \delta 15.5,56.3,107.3,133.8,143.6,158.8,182.4,187.4$.<smiles>CC(=O)OC1=CC(=O)C=C(C)C1=O</smiles>

\section{2-Isopropoxy-6-methylcyclohexa-2,5-diene-1,4-dione (16)}

An adaptation of the procedure reported by Wurm et.al was utilised. ${ }^{4}$ Methoxy-quinone 15 (1.05 g, 6.90 $\mathrm{mmol}$ ) was dissolved in isopropanol $(70 \mathrm{~mL})$ and potassium carbonate $(1.31 \mathrm{~g}, 9.49 \mathrm{mmol})$ added in one portion. The suspension was stirred until TLC showed that no further starting material was present after 6.5 h. The mixture was filtered and the filtrate was partitioned between brine and DCM $(3 \times 30 \mathrm{~mL})$. The 
combined organic extracts were dried over anhydrous $\mathrm{MgSO}_{4}$ and concentrated under reduced pressure. After flash silica gel chromatography (2\% ethyl acetate in DCM), the desired isopropoxy-quinone 16 was obtained as a bright yellow liquid which solidified on standing (0.90 g, 73\%).

m.p. $53-54{ }^{\circ} \mathrm{C}$

${ }^{1} \mathrm{H} \mathrm{NMR}\left(300 \mathrm{MHz}, \mathrm{CDCl}_{3}\right) \delta 1.39(\mathrm{~d}, J=6.2 \mathrm{~Hz}, 6 \mathrm{H}), 2.05(\mathrm{~d}, J=1.7 \mathrm{~Hz}, 3 \mathrm{H}), 4.45(\mathrm{spt}, J=6.0 \mathrm{~Hz}, 1 \mathrm{H}), 5.83(\mathrm{~d}, J$ $=2.4 \mathrm{~Hz}, 1 \mathrm{H}), 6.50-6.52(\mathrm{~m}, 1 \mathrm{H})$.

${ }^{13} \mathrm{C} \mathrm{NMR}\left(100 \mathrm{MHz}, \mathrm{CDCl}_{3}\right) \delta 15.7,21.2,72.4,108.0,133.7,143.8,157.0,182.9,188.0$.

IR (thin film) $v_{\max }: 2980,2920,1678,1649,1595,1312,1230,1190,1104,1053,986,897 \mathrm{~cm}^{-1}$.

HRMS (El+) calculated for $\mathrm{C}_{10} \mathrm{H}_{12} \mathrm{NaO}_{3}: 203.0684$; found: 203.0679 .<smiles>CC(C)OC1=CC(=O)[C@H]2C3C=CC(C3)[C@]2(C)C1=O</smiles>

$\left(1 R^{*}, 4 S^{*}, 4 \mathrm{a} R^{*}, 8 \mathrm{a} S^{*}\right)$-6-Isopropoxy-4a-methyl-1,4,4a,8a-tetrahydro-1,4-methanonaphthalene-5,8-dione (17)

Isopropoxy-quinone $16(0.982 \mathrm{~g}, 5.46 \mathrm{mmol})$ and freshly cracked cyclopentadiene $(3.20 \mathrm{~mL}, 38.1 \mathrm{mmol})$ were stirred in anhydrous DCM over $3 \mathrm{~d}$ or until no starting material was detected by TLC. The solvent and residual diene was removed under reduced pressure, leaving behind the crude product, which was purified by flash silica gel chromatography ( $20 \%$ ethyl acetate in petroleum spirit) to give the product $\mathbf{1 7}$ as a clear liquid (1.26 g, 93\%) which solidified on standing.

m.p. $79-81^{\circ} \mathrm{C}$

${ }^{1} \mathrm{H}$ NMR $\left(400 \mathrm{MHz}, \mathrm{CDCl}_{3}\right) \delta 1.34(\mathrm{~d}, \mathrm{~J}=6.2 \mathrm{~Hz}, 3 \mathrm{H}), 1.35(\mathrm{~d}, \mathrm{~J}=6.2 \mathrm{~Hz}, 3 \mathrm{H}), 1.48(\mathrm{~s}, 3 \mathrm{H}), 1.54(\mathrm{dt}, \mathrm{J}=8.8,1.8$ $\mathrm{Hz}, 1 \mathrm{H}), 1.68(\mathrm{dt}, J=9.2,1.5 \mathrm{~Hz}, 1 \mathrm{H}), 2.83(\mathrm{~d}, J=4.0 \mathrm{~Hz}, 1 \mathrm{H}), 3.10-3.12(\mathrm{~m}, 1 \mathrm{H}), 3.38-3.45(\mathrm{~m}, 1 \mathrm{H}), 4.35$ (sptd, J=6.2, $0.7 \mathrm{~Hz}, 1 \mathrm{H}$ ), $5.86(\mathrm{~s}, 1 \mathrm{H}), 6.03$ (dd, J=5.9, $2.9 \mathrm{~Hz}, 1 \mathrm{H}), 6.13$ (dd, J=5.9, $2.9 \mathrm{~Hz}, 1 \mathrm{H}$ ).

${ }^{13} \mathrm{C} \mathrm{NMR}\left(100 \mathrm{MHz}, \mathrm{CDCl}_{3}\right) \delta 20.93,20.98,26.5,46.4,48.7,52.5,53.8,57.4,72.3,114.6,135.1,137.5,160.7$, $197.8,198.8$.

IR (thin film) $v_{\text {max }}: 2984,1697,1639,1593,1229,1212,1098,1048,1036,865,722 \mathrm{~cm}^{-1}$.

HRMS (El+) calculated for $\mathrm{C}_{15} \mathrm{H}_{18} \mathrm{NaO}_{3}$ : 269.1145; found: 269.1148 .<smiles>CC(C)OC1(C)C(=O)[C@]2(C)CC=C[C@H]1C(=O)C2C</smiles> 
$\left(\left(1 \mathrm{a} R^{*}, 2 \mathrm{a} R^{*}, 3 S^{*}, 6 R^{*}, 6 \mathrm{a} S^{*}, 7 \mathrm{a} S^{*}\right)-1 \mathrm{a}-I s o p r o p o x y-1,1,2 \mathrm{a}-\right.$ trimethyl-1a,2a,3,6,6a,7a-hexahydro-1H-3,6methanocyclopropa[b]naphthalene-2,7-dione (19)

Following the literature procedure, ${ }^{5}$ sodium hydride (60\% dispersion in mineral oil, $0.261 \mathrm{~g}, 2.98 \mathrm{mmol}$ ) in anhydrous DMF $(20 \mathrm{~mL})$ was cooled to $0{ }^{\circ} \mathrm{C}$ using an ice bath under an argon atmosphere. Triisopropylsulfoxonium tetrafluoroborate ${ }^{\text {Errorl Bookmark not defined. }}(0.788 \mathrm{~g}, 2.98 \mathrm{mmol})$ was added in oneportion and stirred for $30 \mathrm{~min}$. Minor effervescence was observed during the deprotonation. The substrate $17(0.605 \mathrm{~g}, 2.46 \mathrm{mmol})$ in anhydrous DMF ( $4 \mathrm{~mL})$ was then added slowly to the sulfoxonium ylid over 10 $\mathrm{min}$. It was crucial to maintain the temperature of the reaction below $5^{\circ} \mathrm{C}$ during the addition. The reaction was stirred for $2 \mathrm{~h}$ at $0{ }^{\circ} \mathrm{C}$ and then warmed gradually to room temperature. Stirring was continued until no starting material was detected by TLC (ca $4 \mathrm{~h}$ ). The dark brown solution was poured onto an ice cold saturated $\mathrm{NH}_{4} \mathrm{Cl}$ solution with caution, and extracted with ethyl acetate $(3 \times 20 \mathrm{~mL})$. The combined organic extracts were washed with brine, dried over anhydrous $\mathrm{Na}_{2} \mathrm{SO}_{4}$ and concentrated under reduced pressure. The residue was purified via flash silica gel chromatography ( $20 \%$ ethyl acetate in petroleum spirit) to give the desired product 19 as a clear oil $(0.50 \mathrm{~g}, 70 \%)$.

${ }^{1} \mathrm{H}$ NMR $\left(300 \mathrm{MHz}, \mathrm{CDCl}_{3}\right) \delta 1.02(\mathrm{~d}, J=6.1 \mathrm{~Hz}, 3 \mathrm{H}), 1.26(\mathrm{~d}, J=6.1 \mathrm{~Hz}, 3 \mathrm{H}), 1.31,(\mathrm{~s}, 3 \mathrm{H}), 1.32(\mathrm{~s}, 3 \mathrm{H}), 1.44$ (dt, J=9.2, $1.6 \mathrm{~Hz}, 1 \mathrm{H}), 1.49(\mathrm{~s}, 3 \mathrm{H}), 1.66(\mathrm{dt}, J=9.2,1.3 \mathrm{~Hz}, 1 \mathrm{H}), 2.14(\mathrm{~s}, 1 \mathrm{H}), 2.59$ (d, J=4.2 Hz, $1 \mathrm{H}), 3.18-$ $3.26(\mathrm{~m}, 1 \mathrm{H}), 3.50$ (qd, J=3.9, $1.9 \mathrm{~Hz}, 1 \mathrm{H}), 3.68(\mathrm{spt}, J=6.2 \mathrm{~Hz}, 1 \mathrm{H}), 6.21-6.26(\mathrm{~m}, 2 \mathrm{H}$ ).

${ }^{13} \mathrm{C} \mathrm{NMR}\left(125 \mathrm{MHz}, \mathrm{CDCl}_{3}\right) \delta 19.5,22.9,23.5,23.7,26.9,33.9,47.3,48.2,51.0,53.1,58.4,59.0,72.6,76.2$, $136.9,137.4,206.3,210.3$.

HRMS (EI+) calculated for $\mathrm{C}_{18} \mathrm{H}_{24} \mathrm{NaO}_{3}$ : 311.1618; found: 311.1630 .<smiles>CC(C)C1(C)C(=O)[C@H](C)C[C@@H](O)[C@H]1C(C)(C)C</smiles><smiles>CC(C)[C@@]1(O)[C@@H](O)[C@@H](C)[C@H](O)C[C@H]1C</smiles>

$\left(1 S^{*}, 3 R^{*}\right)$-5-Hydroxy-1-isopropoxy-3,7,7-trimethylbicyclo[4.1.0]heptan-2-one (22)

The retro-Diels Alder reaction was carried out in a Kulgehrohr distillation apparatus by heating the cyclopropyl adduct $19\left(1.82 \mathrm{~g}, 6.31 \mathrm{mmol}\right.$ ) to $140{ }^{\circ} \mathrm{C}$ at $520-570 \mathrm{mmHg}$ (Büchi vacuum controller). The reaction was monitored by TLC, which showed no starting material after $2 \mathrm{~h}$. The bright yellow liquid, containing relatively pure endione $\mathbf{2 0}$, was taken up in methanol $(25 \mathrm{~mL})$ and cooled to $0{ }^{\circ} \mathrm{C}$. To this was added $\mathrm{NaBH}_{4}(0.70 \mathrm{~g}, 5.3 \mathrm{mmol})$ in small portions over $2 \mathrm{~min}$. The reaction was stirred for $2 \mathrm{~h}$ while the bath temperature warmed to $6{ }^{\circ} \mathrm{C}$. TLC indicated endione $\mathbf{2 0}$ was fully consumed. The reaction was quenched by adding a cold solution of saturated $\mathrm{NH}_{4} \mathrm{Cl}$. To assist with the extraction process, the methanol was partially removed under reduced pressure. The residue was then partitioned with water and ethyl acetate $(3 \times 15 \mathrm{~mL})$. The organic extracts were combined and washed with brine. After drying the organic layer over anhydrous $\mathrm{Na}_{2} \mathrm{SO}_{4}$, the solvent was removed via rotary evaporation. The yellow residue was purified by flash silica gel chromatography $(40 \%$ ethyl acetate in petroleum spirit) affording two diastereomers $\left(5 R^{*}\right)$-22 and $\left(5 S^{*}\right)$-22 (1.47 g, 92\% over 2 steps, dr 2:3), as a colourless oil, that were collected as a mixture. The two diastereomers were separated for characterisation purposes.

$\left(5 R^{*}\right)-22$ 
${ }^{1} \mathrm{H}$ NMR $\left(500 \mathrm{MHz}, \mathrm{CDCl}_{3}\right) \delta 1.03(\mathrm{~s}, 3 \mathrm{H}), 1.10(\mathrm{~d}, \mathrm{~J}=6.6 \mathrm{~Hz}, 3 \mathrm{H}), 1.15(\mathrm{~d}, \mathrm{J=6.2} \mathrm{Hz}, 3 \mathrm{H}), 1.16(\mathrm{~d}, \mathrm{~J}=6.2 \mathrm{~Hz}, 3$ H), $1.23(\mathrm{~s}, 3 \mathrm{H}$ ), 1.46 (t, J=2.2 Hz, $1 \mathrm{H}$ ), 1.73 (ddd, J=15.8, 12.8, 3.7 Hz, $1 \mathrm{H}$ ), 1.76 (br. s, $1 \mathrm{H}$ ), 2.00 (ddt, $J=15.1,6.6,2.2 \mathrm{~Hz}, 1 \mathrm{H}), 2.65$ (dt, J=12.9, $6.6 \mathrm{~Hz}, 1 \mathrm{H}$ ), 3.71 (spt, J=6.2 Hz, $1 \mathrm{H}$ ), 4.43 (br. s, $1 \mathrm{H}$ ).

${ }^{13} \mathrm{CNMR}\left(100 \mathrm{MHz}, \mathrm{CDCl}_{3}\right) \delta 13.7,17.9,23.0,23.2,24.6,27.3,36.7,38.66,38.73,63.9,70.2,72.6,211.1$.

$\left(5 S^{*}\right)-22$

${ }^{1} \mathrm{H}$ NMR $\left(500 \mathrm{MHz}, \mathrm{CDCl}_{3}\right) \delta 1.12(\mathrm{~d}, J=6.2 \mathrm{~Hz}, 3 \mathrm{H}), 1.12(\mathrm{~d}, J=6.2 \mathrm{~Hz}, 3 \mathrm{H}), 1.13(\mathrm{~d}, J=6.2 \mathrm{~Hz}, 3 \mathrm{H}), 1.71$ (br. s, $1 \mathrm{H}), 1.20(\mathrm{~s}, 3 \mathrm{H}), 1.24(\mathrm{~s}, 3 \mathrm{H}), 1.55-1.64(\mathrm{~m}, 2 \mathrm{H}), 2.15-2.30(\mathrm{~m}, 1 \mathrm{H}), 2.25(\mathrm{tt}, J=12.8,6.6 \mathrm{~Hz}, 1 \mathrm{H}), 3.62$ (spt, J=6.2 Hz, $1 \mathrm{H}$ ), 4.65 (ddd, J=11.3, 6.9, $5.7 \mathrm{~Hz}, 1 \mathrm{H}$ ).

${ }^{13} \mathrm{CNMR}\left(100 \mathrm{MHz}, \mathrm{CDCl}_{3}\right) \delta 14.1,19.0,22.8,23.2,24.8,28.2,36.7,37.5,42.7,66.5,71.7,72.0,209.6$.

HRMS (EI+) calculated for $\mathrm{C}_{13} \mathrm{H}_{22} \mathrm{Na} \mathrm{O}_{3}: 249.1467$; found: 249.1461.<smiles>CC(C)C1(O)C(O)[C@H](C)C[C@@H](O)C1C(C)(C)C</smiles>

$\left(1 R^{*}, 2 R^{*}, 3 S^{*}\right)$-1-Isopropoxy-3,7,7-trimethylbicyclo[4.1.0]heptane-2,5-diol (23)

A diastereomeric mixture of $(R)$ - and $(S)$-ketone $22(1.40 \mathrm{~g}, 6.18 \mathrm{mmol})$ in anhydrous THF $(20 \mathrm{~mL})$ was cooled to $0{ }^{\circ} \mathrm{C}$ under an argon atmosphere. To this was added DIBAL-H (1.0 M in hexane, $18.5 \mathrm{~mL}$ ) dropwise over $15 \mathrm{~min}$. The reaction was stirred for $2 \mathrm{~h}$ while the bath temperature was gradually warmed to $10^{\circ} \mathrm{C}$, at which no starting material was detected by TLC. On re-cooling to $0{ }^{\circ} \mathrm{C}$, a saturated potassium sodium tartrate (Rochelle's salt) solution was added. A gel-like material was formed initially. The heterogeneous solution was stirred for $1 \mathrm{~h}$ at the same temperature and then at room temperature until all gel-like material redissolved. The solution was extracted with ethyl acetate $(3 \times 25 \mathrm{~mL})$. The combined organic extracts were washed with brine, dried over anhydrous $\mathrm{Na}_{2} \mathrm{SO}_{4}$ and concentrated under reduced pressure. The crude residue was subjected to flash silica gel chromatography ( $40 \%$ ethyl acetate in petroleum spirit) giving the diol as mainly two diastereomers $(1.20 \mathrm{~g}, 85 \%$, colourless oil), collected as one fraction. Separation of the diastereomers by flash chromatography was attempted for characterisation purposes.

$\left(5 R^{*}\right)-23$ (off-white solid)

m.p. $79-81^{\circ} \mathrm{C}$

${ }^{1} \mathrm{H}$ NMR $\left(500 \mathrm{MHz}, \mathrm{CDCl}_{3}\right) \delta 0.99(\mathrm{~s}, 3 \mathrm{H}), 1.03(\mathrm{~d}, \mathrm{~J}=6.6 \mathrm{~Hz}, 3 \mathrm{H}), 1.16(\mathrm{~s}, 3 \mathrm{H}), 1.18(\mathrm{~d}, \mathrm{~J}=6.2 \mathrm{~Hz}, 3 \mathrm{H}), 1.15-$ $1.21(\mathrm{~m}, 2 \mathrm{H}), 1.31$ (d, J=6.2 Hz, $3 \mathrm{H}$ ), 1.57 (ddt, J=15.4, 3.7, 1.8, $1.8 \mathrm{~Hz}, 1 \mathrm{H}$ ), 1.63 (br. s, $1 \mathrm{H}), 1.74-1.85$ (m, $1 \mathrm{H}), 3.16$ (d, J=10.6 Hz, 1 H), 3.24 (br. s, 1 H), 3.78 (spt, J=6.2 Hz, 1 H), 4.01 (dd, J=2.6, $1.8 \mathrm{~Hz}, 1 \mathrm{H}$ ).

${ }^{13} \mathrm{C} \mathrm{NMR}\left(100 \mathrm{MHz}, \mathrm{CDCl}_{3}\right) \delta 16.1,18.3,22.8,23.2,23.5,24.4,31.4,32.8,39.7,63.9,66.2,70.3,72.5$.

IR (thin film) $v_{\text {max }}: 3436,2922,1381,1368,1154,1131,1110,1095,1089,1013,958,932,839 \mathrm{~cm}^{-1}$.

$\left(5 S^{*}\right)-23$ [colourless oil, the sample was mixed with a small amount of $\left(5 R^{*}\right)-23$ ] 
${ }^{1} \mathrm{H} \mathrm{NMR}\left(400 \mathrm{MHz}, \mathrm{CDCl}_{3}\right) \delta 1.06(\mathrm{~d}, J=6.5 \mathrm{~Hz}, 3 \mathrm{H}), 1.16-1.19(\mathrm{~m}, 1 \mathrm{H}), 1.18(\mathrm{~d}, J=6.2 \mathrm{~Hz}, 3 \mathrm{H}), 1.19(\mathrm{~s}, 3 \mathrm{H})$, $1.21(\mathrm{~s}, 3 \mathrm{H}), 1.23(\mathrm{~d}, J=6.2 \mathrm{~Hz}, 3 \mathrm{H}), 1.31(\mathrm{~d}, J=6.2 \mathrm{~Hz}, 1 \mathrm{H}), 1.40(\mathrm{~d}, J=7.3 \mathrm{~Hz}, 1 \mathrm{H}), 1.43-1.54(\mathrm{~m}, 2 \mathrm{H}), 1.84$ (ddd, J=13.5, 7.5, 2.8 Hz, 1 H), 3.29 (d, J=10.6 Hz, 1 H), 3.68 (spt, J=6.2 Hz, 1 H), 4.38 (dt, J=10.9, $7.4 \mathrm{~Hz}, 1 \mathrm{H}$ ).

${ }^{13} \mathrm{CNMR}\left(100 \mathrm{MHz}, \mathrm{CDCl}_{3}\right) \delta 17.5,18.3,23.2,23.4,23.5,26.9,31.3,36.8,38.9,67.7,69.4,70.1,72.1$.

HRMS (El+) calculated for $\mathrm{C}_{13} \mathrm{H}_{24} \mathrm{NaO}_{3}: 251.1623$; found: 251.1618 .

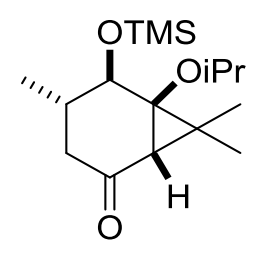

$\left(1 S^{*}, 4 S^{*}, 5 R^{*}, 6 R^{*}\right)-6$-Isopropoxy-4,7,7-trimethyl-5-((trimethylsilyl)oxy)bicyclo[4.1.0]heptan-2-one (24)

Under an argon atmosphere, diol $23(1.18 \mathrm{~g}, 5.17 \mathrm{mmol})$ in anhydrous DCM $(8 \mathrm{~mL})$ was cooled to $0{ }^{\circ} \mathrm{C}$. Triethylamine $(1.3 \mathrm{~mL}, 8.4 \mathrm{mmol})$ was added, followed by TMSCl $(0.60 \mathrm{~mL}, 4.8 \mathrm{mmol})$. The reaction was left to stir while the temperature increased gradually to room temperature. No starting material was observed by TLC after $15 \mathrm{~h}$. The suspension was poured into a cold saturated $\mathrm{NaHCO}_{3}$ solution, followed by extraction with DCM $(3 \times 20 \mathrm{~mL})$. The combined organic extracts were washed with brine, dried over anhydrous $\mathrm{Na}_{2} \mathrm{SO}_{4}$ and then concentrated under reduced pressure. The crude material was taken up in methanol (24 $\mathrm{mL}$ ) and cooled to $0{ }^{\circ} \mathrm{C}$. Citric acid $(29 \mathrm{mg}, 0.15 \mathrm{mmol})$ in methanol $(1 \mathrm{~mL})$ was then added. The reaction was closely followed by TLC until the mono-deprotection was completed. While still maintaining the same temperature, a saturated $\mathrm{NaHCO}_{3}$ solution was added and subsequently approximately half of the methanol was removed under reduced pressure. The remaining solution was partitioned with water and ethyl acetate $(3 \times 20 \mathrm{~mL})$. The combined organic extracts were washed with brine, dried over anhydrous $\mathrm{Na}_{2} \mathrm{SO}_{4}$, and concentrated under reduced pressure. The crude oil was redissolved in anhydrous $\mathrm{DCM}(10 \mathrm{~mL})$ was added $N$-methylmorpholine $N$-oxide $(0.95 \mathrm{~g}, 8.1 \mathrm{mmol})$, TPAP $(71 \mathrm{mg}, 0.20 \mathrm{mmol})$ and a spatula of $4 \AA$ molecular sieves powder respectively. The dark green suspension was stirred for $3 \mathrm{~h}$, at which point no starting material could be detected by TLC. The reaction mixture was passed through a plug of silica gel. The plug was then rinsed with freshly distilled DCM. The combined DCM solutions were dried over anhydrous $\mathrm{Na}_{2} \mathrm{SO}_{4}$ and concentrated under reduced pressure to give the desired ketone $\mathbf{2 4}$ as a colourless oil ( $0.75 \mathrm{~g}, 66 \%$ over 3 steps). Ketone $\mathbf{2 4}$ was used in the following reaction without further purification.

${ }^{1} \mathrm{H}$ NMR $\left(400 \mathrm{MHz}, \mathrm{CDCl}_{3}\right.$ ) $\delta 0.15(\mathrm{~s}, 9 \mathrm{H}), 1.00$ (d, J=6.2 Hz, $\left.3 \mathrm{H}\right), 1.07$ (d, J=6.2 Hz, $3 \mathrm{H}$ ), $1.18(\mathrm{~s}, 3 \mathrm{H}), 1.22$ (d, $J=6.2 \mathrm{~Hz}, 3 \mathrm{H}), 1.28(\mathrm{~s}, 3 \mathrm{H}), 1.69$ (dd, J=17.5, $13.5 \mathrm{~Hz}, 1 \mathrm{H}), 1.86(\mathrm{dd}, J=2.1,0.6 \mathrm{~Hz}, 1 \mathrm{H}), 2.04-2.18(\mathrm{~m}, 1 \mathrm{H})$, 2.37 (ddd, J=17.5, 4.0, $1.9 \mathrm{~Hz}, 1 \mathrm{H}$ ), 3.60 (d, J=10.9 Hz, $1 \mathrm{H}$ ), 3.99 (spt, J=6.2 Hz, $1 \mathrm{H}$ ).

${ }^{13} \mathrm{CNMR}\left(100 \mathrm{MHz}, \mathrm{CDCl}_{3}\right) \delta-0.2(3 \mathrm{C}), 17.9,18.3,22.8,23.5,23.8,34.5,39.4,42.3,47.2,71.6,71.9,72.2$, 207.2.

IR (thin film) $v_{\max }: 2960,1680,1250,1159,1102,1084,1066,928,890,862,839 \mathrm{~cm}^{-1}$.

HRMS (EI+) calculated for $\mathrm{C}_{16} \mathrm{H}_{30} \mathrm{NaO}_{3} \mathrm{Si}$ : 321.1862; found: 321.1856 . 


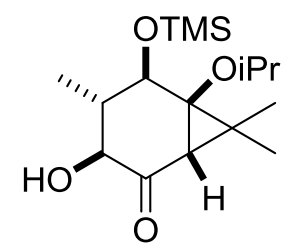

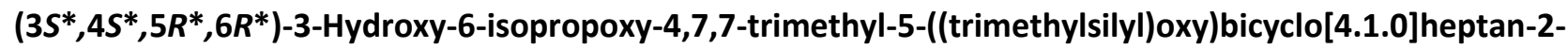
one (26)

In a round bottom flask, LDA was freshly prepared by slowly adding ${ }^{\mathrm{n}} \mathrm{BuLi}(1.95 \mathrm{M}$ in cyclohexane, $1.6 \mathrm{~mL}$, $3.0 \mathrm{mmol}$ ) to $\mathrm{N}, \mathrm{N}$-diisopropylamine $(0.46 \mathrm{~mL}, 3.3 \mathrm{mmol})$ in anhydrous THF $(8 \mathrm{~mL})$ at $0{ }^{\circ} \mathrm{C}$ under an argon atmosphere. After stirring at the same temperature for $20 \mathrm{~min}$, the colourless LDA solution was added dropwise to ketone $24(0.75 \mathrm{~g}, 2.5 \mathrm{mmol})$ in anhydrous THF $(10 \mathrm{~mL})$ precooled to $-78{ }^{\circ} \mathrm{C}$ under an argon atmosphere. Once the addition was completed, the reaction was stirred for $30 \mathrm{~min}$ before neat TMSCl (freshly distilled from $\mathrm{CaH}_{2}, 0.38 \mathrm{~mL}, 3.0 \mathrm{mmol}$ ) was added slowly, which formed a white precipitate over time. The reaction was followed by TLC and stirred until all the starting material was consumed. Quenching of the reaction was performed by pouring the suspension into cold aqueous $\mathrm{NaHCO}_{3}$ solution and extracted with diethyl ether $(3 \times 15 \mathrm{~mL})$. The combined ether extracts were washed with brine, dried over anhydrous $\mathrm{Na}_{2} \mathrm{SO}_{4}$ and concentrated under reduced pressure to give the crude TMS enol ether $\mathbf{2 5}$. The crude $\mathbf{2 5}$ was redissolved in acetone $\left(3 \mathrm{~mL}\right.$ ) and cooled to $0{ }^{\circ} \mathrm{C}$. DMDO solution ${ }^{6}$ (approx. $0.05 \mathrm{M}$ in acetone) was added to the acetone solution of 25 in $3 \mathrm{~mL}$ portions with a minute of stirring in between each addition. Once $40 \mathrm{~mL}$ of the DMDO solution was added, the reaction was then stirred for $20 \mathrm{~min}$ before another 3 portions of DMDO was added. The temperature of the reaction was maintained at $0{ }^{\circ} \mathrm{C}$ the entire time. After stirring for another $45 \mathrm{~min}$, the reaction was stirred for an extra $15 \mathrm{~min}$ at room temperature before concentrating under reduced pressure. The residue was partitioned between a saturated aqueous $\mathrm{NH}_{4} \mathrm{Cl}$ solution and diethyl ether $(3 \times 15 \mathrm{~mL})$. The combined ether extracts were dried with anhydrous $\mathrm{Na}_{2} \mathrm{SO}_{4}$, followed by concentration under reduced pressure. The crude product was subjected to flash silica gel chromatography (15\% ethyl acetate in petroleum spirit), which afforded the desired hydroxyl-ketone $\mathbf{2 6}(0.38 \mathrm{~g}, 49 \%$ over 2 steps, colourless oil) was obtained and starting ketone $\mathbf{2 4}$ (0.22 g, 29\%) were recovered.

${ }^{1} \mathrm{H} \mathrm{NMR}\left(400 \mathrm{MHz}, \mathrm{CDCl}_{3}\right) \delta 0.16(\mathrm{~s}, 9 \mathrm{H}), 1.07$ (d, J=6.2 Hz, $\left.3 \mathrm{H}\right), 1.17$ (d, J=6.5 Hz, $\left.3 \mathrm{H}\right), 1.20$ (d, J=6.2 Hz, 3 H), $1.22(\mathrm{~s}, 3 \mathrm{H}), 1.31(\mathrm{~s}, 3 \mathrm{H}), 1.93(\mathrm{tq}, J=11.2,6.2 \mathrm{~Hz}, 1 \mathrm{H}), 2.08(\mathrm{~s}, 1 \mathrm{H}), 3.40$ (dd, J=12.3, $2.3 \mathrm{~Hz}, 1 \mathrm{H}), 3.68$ (d, J=11.2 Hz, $1 \mathrm{H}$ ), 3.88 (d, J=2.6 Hz, $1 \mathrm{H}), 4.00$ (spt, J=6.2 Hz, $1 \mathrm{H}$ ).

${ }^{13} \mathrm{CNMR}\left(100 \mathrm{MHz}, \mathrm{CDCl}_{3}\right) \delta-0.2(3 \mathrm{C}), 15.3,17.9,23.0,23.3,23.7,36.1,42.7,48.5,70.0,72.2,75.2,77.1$, 206.7.

IR (thin film) $v_{\max }: 3436,2922,1381,1368,1154,1131,1110,1080,1013,958,932,839 \mathrm{~cm}^{-1}$.

HRMS (EI+) calculated for $\mathrm{C}_{16} \mathrm{H}_{30} \mathrm{NaO}_{4} \mathrm{Si}$ : 337.1811; found: 337.1806 .

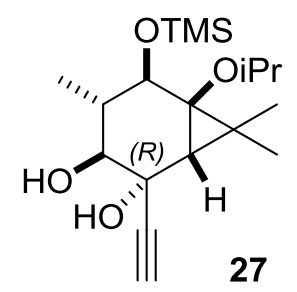

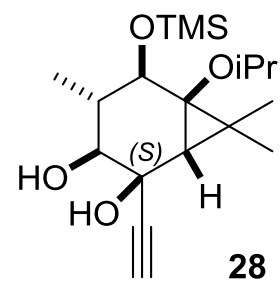


At ambient temperature, commercially available ethynyl magnesium bromide solution (Sigma-Aldrich, 0.5 $\mathrm{M}$ in THF, $5.1 \mathrm{~mL}$ ) was added slowly in one-portion to a solution of hydroxy-ketone $26(0.20 \mathrm{~g}, 0.64 \mathrm{mmol})$ in anhydrous THF $(3 \mathrm{~mL})$ under an argon atmosphere. The orange solution was stirred for $24 \mathrm{~h}$ at which point no starting material was detected by TLC. The reaction was quenched by pouring the mixture into a cold saturated $\mathrm{NH}_{4} \mathrm{Cl}$ solution, which was extracted with ethyl acetate $(3 \times 10 \mathrm{~mL})$. The combined organic extracts were washed with brine, dried over anhydrous $\mathrm{Na}_{2} \mathrm{SO}_{4}$ and concentrated under reduced pressure. The crude product was subjected to flash silica gel chromatography (20\% ethyl acetate in petroleum spirit), which gave diols 27 (73 mg, 34\%) and 28 (87 mg, 40\%) in two fractions.

27 (colourless oil)

${ }^{1} \mathrm{H}$ NMR $\left(500 \mathrm{MHz}, \mathrm{C}_{6} \mathrm{D}_{6}\right) \delta 0.18(\mathrm{~s}, 9 \mathrm{H}), 1.06(\mathrm{~s}, 3 \mathrm{H}), 1.15(\mathrm{~d}, J=6.6 \mathrm{~Hz}, 3 \mathrm{H}), 1.18(\mathrm{~d}, J=6.2 \mathrm{~Hz}, 3 \mathrm{H}), 1.24(\mathrm{~s}, 3$ H), 1.34 (d, J=6.2 Hz, $3 \mathrm{H}$ ), 1.70 (d, J=9.5 Hz, $1 \mathrm{H}$ ), 1.77 (s, $1 \mathrm{H}), 1.93$ (tq, J=11.0, $6.2 \mathrm{~Hz}, 1 \mathrm{H}), 2.01(\mathrm{~s}, 1 \mathrm{H}$ ), 2.52 (br. s, 1 H), 3.03 (dd, J=11.4, $9.5 \mathrm{~Hz}, 1 \mathrm{H}$ ), 3.34 (d, J=10.6 Hz, 1 H), 3.98 (spt, J=6.2 Hz, 1 H).

${ }^{13} \mathrm{C}$ NMR $\left(100 \mathrm{MHz}, \mathrm{C}_{6} \mathrm{D}_{6}\right) \delta 0.4(3 \mathrm{C}), 14.7,18.0,24.1,24.5,24.6,28.8,38.6,43.6,68.2,72.48,72.54,74.4$, 74.7, 78.9, 86.0.

IR (thin film) $v_{\text {max }}: 3461,3310,1260,1249,1142,1101,1048,1023,876,839, \mathrm{~cm}^{-1}$.

28 (off-white solid)

m.p. $141.5-143.0^{\circ} \mathrm{C}$

${ }^{1} \mathrm{H}$ NMR $\left(500 \mathrm{MHz}, \mathrm{C}_{6} \mathrm{D}_{6}\right) \delta 0.20(\mathrm{~s}, 9 \mathrm{H}), 1.03(\mathrm{~d}, J=5.9 \mathrm{~Hz}, 3 \mathrm{H}), 1.04(\mathrm{~s}, 3 \mathrm{H}), 1.15$ (d, J=6.2 Hz, $\left.3 \mathrm{H}\right), 1.29(\mathrm{~s}, 3$ H), $1.30(\mathrm{~d}, J=5.9 \mathrm{~Hz}, 3 \mathrm{H}), 1.70(\mathrm{~s}, 1 \mathrm{H}), 1.71(\mathrm{~d}, J=5.5 \mathrm{~Hz}, 1 \mathrm{H}), 1.98(\mathrm{~s}, 1 \mathrm{H}), 1.99$ (tq, J=11.0, $6.6 \mathrm{~Hz}, 1 \mathrm{H})$, $2.86(\mathrm{dd}, J=11.2,5.7 \mathrm{~Hz}, 1 \mathrm{H}), 3.22(\mathrm{~d}, J=11.0 \mathrm{~Hz}, 1 \mathrm{H}), 3.28(\mathrm{~s}, 1 \mathrm{H}), 4.02(\mathrm{spt}, J=6.2 \mathrm{~Hz}, 1 \mathrm{H})$.

${ }^{13} \mathrm{C} N M R\left(100 \mathrm{MHz}, \mathrm{C}_{6} \mathrm{D}_{6}\right) \delta 0.4(3 \mathrm{C}), 14.7,17.5,23.8,23.9,24.5,25.2,38.4,39.0,64.7,67.8,71.2,71.5,72.5$, 78.7, 87.7.

HRMS (EI+) calculated for $\mathrm{C}_{18} \mathrm{H}_{32} \mathrm{NaO}_{4} \mathrm{Si}: 363.1968$; found: 363.1962 .<smiles>COC1[C@H](C)c2occc2[C@H]2[C@H]1C2(C)C(C)C</smiles>

$\left(\left(\left(4 R^{*}, 5 R^{*}, 5 a R^{*}, 6 a R^{*}\right)-5 a-I s o p r o p o x y-4,6,6\right.\right.$-trimethyl-5,5a,6,6a-tetrahydro-4H-cyclopropa[e]benzofuran5-yl)oxy)trimethylsilane (29)

A modified procedure of that reported by Aponick et.al. ${ }^{7}$ was used. A gold(I) chloride solution was freshly prepared by stirring commercially available gold $(\mathrm{I})$ chloride $(1.1 \mathrm{mg}, 4.7 \mu \mathrm{mol})$ in anhydrous THF $(1 \mathrm{~mL})$ under an argon atmosphere. After a few minutes, the undissolved catalyst was allowed to settle and only the yellow solution was used in the subsequent reaction. 
To 27 (38 $\mathrm{mg}, 0.11 \mathrm{mmol}$ ) in anhydrous THF $(4 \mathrm{~mL})$ under an atmosphere of argon was added the catalyst solution above $(0.8 \mathrm{~mL})$. The reaction was left to stir in the dark and was monitored closely by TLC. The colour of the reaction intensified gradually and turned to a deep pink. Upon completion of reaction, the solvent was immediately removed under in vacuo under ambient temperature. The residue was redissolved in a small amount of petroleum spirit and passed through a plug of neutral aluminium oxide (petroleum spirit) to give the desired furan 29 as a colourless liquid (29 mg, 80\%). [Notes: 1) Fresh catalyst is essential; 2) The product is highly sensitive to acid and base.]

${ }^{1} \mathrm{H}$ NMR $\left(500 \mathrm{MHz}, \mathrm{C}_{6} \mathrm{D}_{6}\right) \delta 0.20(\mathrm{~s}, 9 \mathrm{H}), 0.82(\mathrm{~s}, 3 \mathrm{H}), 1.04(\mathrm{~d}, J=6.2 \mathrm{~Hz}, 3 \mathrm{H}), 1.27(\mathrm{~d}, J=6.2 \mathrm{~Hz}, 3 \mathrm{H}), 1.40(\mathrm{~s}, 3$ H), 1.44 (d, J=7.0 Hz, 3 H), 1.72 (d, J=1.1 Hz, 1 H), 2.99 (dqt, J=9.9, 6.6, $1.5 \mathrm{~Hz}, 1 \mathrm{H}$ ), 3.45 (d, J=9.9 Hz, $1 \mathrm{H}$ ), 3.96 (spt, J=6.2 Hz, $1 \mathrm{H}$ ), 6.09 (d, J=2.0 Hz, $1 \mathrm{H}$ ), 7.09 (dd, J=2.0, $1.1 \mathrm{~Hz}, 1 \mathrm{H}$ ).

${ }^{13} \mathrm{C}$ NMR $\left(100 \mathrm{MHz}, \mathrm{C}_{6} \mathrm{D}_{6}\right) \delta 0.4(3 \mathrm{C}), 14.5,17.3,22.3,23.9,24.4,27.0,31.4,37.5,65.6,71.8,75.1,111.4$, $114.3,141.9,154.8$.

HRMS (El+) calculated for $\mathrm{C}_{18} \mathrm{H}_{30} \mathrm{NaO}_{3} \mathrm{Si}$ : 345.1862; found: 345.1856 .

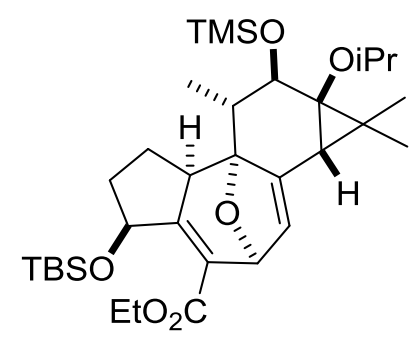

Ethyl $\quad\left(1 a R^{*}, 3 S^{*}, 5 S^{*}, 7 a R^{*}, 7 b R^{*}, 8 R^{*}, 9 R^{*}, 9 a R^{*}\right)-5$-((tert-butyldimethylsilyl)oxy)-9a-isopropoxy-1,1,8trimethyl-9-((trimethylsilyl)oxy)-1,1a,3,5,6,7,7a,8,9,9a-decahydro-3,7b-epoxycyclopropa[3,4]benzo[1,2e]azulene-4-carboxylate (30)

Furan $29(29 \mathrm{mg}, 90 \mu \mathrm{mol})$ and $\mathrm{Rh}_{2}(\mathrm{OAc})_{4}(0.7 \mathrm{mg}, 1.6 \mu \mathrm{mol})$ were mixed in anhydrous cyclohexane $(0.3 \mathrm{~mL})$ and heated to $60^{\circ} \mathrm{C}$ under an argon atmosphere. The solubility of the catalyst increased as the temperature increased. The diazo compound $9^{8}(39 \mathrm{mg}, 0.13 \mathrm{mmol})$ in anhydrous cyclohexane $(0.4 \mathrm{~mL})$ was added dropwise to the hot solution. Once the addition was completed, the reaction was stirred for $1 \mathrm{~h}$. Upon cooling to ambient temperature, the solution was passed through a plug of neutral alumina ( $20 \%$ ethyl acetate in petroleum spirit). After removal of solvent, the residue was subjected to further chromatography using neutral alumina ( $5 \%$ ethyl acetate in petroleum spirit) to give the desired product $\mathbf{3 0}$ as a slightly yellow resinous oil (36 $\mathrm{mg}, 66 \%)$.

${ }^{1} \mathrm{H}$ NMR $\left(500 \mathrm{MHz}, \mathrm{C}_{6} \mathrm{D}_{6}\right) \delta 0.14(\mathrm{~s}, 3 \mathrm{H}), 0.21(\mathrm{~s}, 9 \mathrm{H}), 0.27(\mathrm{~s}, 3 \mathrm{H}), 0.94(\mathrm{~s}, 9 \mathrm{H}), 0.98(\mathrm{~s}, 3 \mathrm{H}), 0.99(\mathrm{t}, J=7.0$ $\mathrm{Hz}, 3 \mathrm{H}), 1.04$ (d, J=5.9 Hz, $3 \mathrm{H}), 1.26(\mathrm{~s}, 3 \mathrm{H}), 1.30-1.43(\mathrm{~m}, 3 \mathrm{H}), 1.38(\mathrm{~d}, J=6.2 \mathrm{~Hz}, 3 \mathrm{H}), 1.40$ (d, J=7.0 Hz, 3 H), 1.58 (dt, J=10.5, $5.2 \mathrm{~Hz}, 1 \mathrm{H}$ ), 1.70 (dd, J=12.5, $5.1 \mathrm{~Hz}, 1 \mathrm{H}$ ), 2.40 (dq, J=11.4, $6.8 \mathrm{~Hz}, 1 \mathrm{H}$ ), 2.75 (dd, J=12.7, $6.4 \mathrm{~Hz}, 1 \mathrm{H}$ ), 3.37 (d, J=11.4 Hz, $1 \mathrm{H}$ ), 3.99 (spt, J=6.2 Hz, $1 \mathrm{H}$ ), 4.01 (dq, J=10.6, 7.0 Hz, $1 \mathrm{H}$ ), 4.12 (dq, J=10.6, $7.0 \mathrm{~Hz}, 1 \mathrm{H}), 5.08(\mathrm{~d}, J=2.2 \mathrm{~Hz}, 1 \mathrm{H}), 5.18(\mathrm{~d}, J=5.1 \mathrm{~Hz}, 1 \mathrm{H}), 6.37$ (t, J=2.4 Hz, $1 \mathrm{H}$ ).

${ }^{13} \mathrm{CNMR}\left(100 \mathrm{MHz}, \mathrm{C}_{6} \mathrm{D}_{6}\right) \delta-4.4,-3.5,0.6$ (3C), 11.7, 14.7, 18.7, 18.8, 23.0, 24.6, 24.7, 25.2, 26.0, 26.55 (3C), $26.64,36.0,43.3,46.8,60.2,68.5,70.3,72.0,72.1,77.7,90.1,132.1,136.6,139.6,155.7,165.6$.

HRMS (El+) calculated for $\mathrm{C}_{33} \mathrm{H}_{56} \mathrm{NaO}_{6} \mathrm{Si}_{2}$ : 627.3513; found: 627.3508 . 


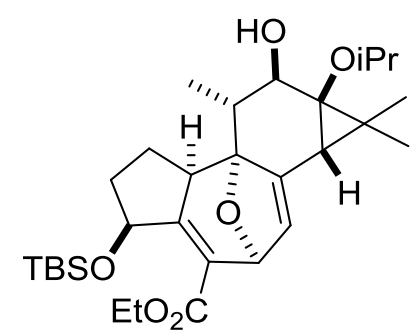

Ethyl $\left(1 a R^{*}, 3 S^{*}, 5 S^{*}, 7 a R^{*}, 7 b R^{*}, 8 R^{*}, 9 R^{*}, 9 a R^{*}\right)-5$-((tert-butyldimethylsilyl)oxy)-9-hydroxy-9a-isopropoxy1,1,8-trimethyl-1,1a,3,5,6,7,7a,8,9,9a-decahydro-3,7b-epoxycyclopropa[3,4]benzo[1,2-e]azulene-4carboxylate (31)

Compound $30(8.0 \mathrm{mg}, 13 \mu \mathrm{mol})$ was dissolved in methanol $(1.2 \mathrm{~mL})$ and stirred with a catalytic amount of Amberlyst I 15 for $2.5 \mathrm{~h}$ at which point no starting material could be detected by TLC. The resin was filtered off and rinsed with ethyl acetate. The combined filtrates were concentrated in vacuo and the residue purified via chromatography with neutral alumina ( $15 \%$ ethyl acetate in petroleum spirit) to give the desired product $31(6.0 \mathrm{mg}, 85 \%)$ as a colourless oil.

${ }^{1} \mathrm{H}$ NMR $\left(500 \mathrm{MHz}, \mathrm{C}_{6} \mathrm{D}_{6}\right) \delta 0.15(\mathrm{~s}, 3 \mathrm{H}), 0.28(\mathrm{~s}, 3 \mathrm{H}), 0.89(\mathrm{~d}, J=5.5 \mathrm{~Hz}, 3 \mathrm{H}), 0.90(\mathrm{~s}, 3 \mathrm{H}), 0.94(\mathrm{~s}, 9 \mathrm{H}), 0.99$ (t, J=7.2 Hz, $3 \mathrm{H}), 1.15(\mathrm{~s}, 3 \mathrm{H}), 1.16(\mathrm{~d}, J=7.0 \mathrm{~Hz}, 3 \mathrm{H}), 1.31-1.38(\mathrm{~m}, 2 \mathrm{H}), 1.44(\mathrm{~d}, J=2.2 \mathrm{~Hz}, 1 \mathrm{H}), 1.47-1.52$ $(\mathrm{m}, 1 \mathrm{H}), 1.59(\mathrm{~d}, J=7.0 \mathrm{~Hz}, 3 \mathrm{H}), 1.68-1.72(\mathrm{~m}, 1 \mathrm{H}), 2.17(\mathrm{dq}, J=12.1,7.0 \mathrm{~Hz}, 1 \mathrm{H}), 2.71-2.78(\mathrm{~m}, 1 \mathrm{H}), 2.83$ (d, J=6.2 Hz, $1 \mathrm{H}$ ), 3.38 (dd, J=11.9, $6.4 \mathrm{~Hz}, 1 \mathrm{H}$ ), 3.75 (spt, J=6.2 Hz, $1 \mathrm{H}$ ), $4.02(\mathrm{dq}, J=10.6,7.0 \mathrm{~Hz}, 1 \mathrm{H}), 4.11$ (dq, J=10.6, 7.0 Hz, $1 \mathrm{H}$ ), $5.04(\mathrm{~d}, J=2.2 \mathrm{~Hz}, 1 \mathrm{H}), 5.15-5.20(\mathrm{~m}, 1 \mathrm{H}), 6.35$ (t, J=2.4 Hz, $1 \mathrm{H}$ ).

${ }^{13} \mathrm{C}$ NMR $\left(125 \mathrm{MHz}, \mathrm{C}_{6} \mathrm{D}_{6}\right) \delta-4.5,-3.5,11.1,14.7,18.2,18.7,22.4,24.3,24.5,25.2,25.5,26.6(3 \mathrm{C}), 27.3,36.1$, $43.1,46.5,60.3,68.9,70.9,71.9,73.6,77.7,89.9,132.1,136.0,140.2,155.6,165.5$.

HRMS (EI+) calculated for $\mathrm{C}_{33} \mathrm{H}_{56} \mathrm{NaO}_{6} \mathrm{Si}_{2}$ : 627.3513; found: 627.3508 .

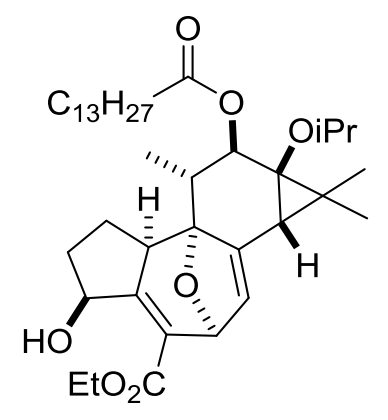

Ethyl

$\left(1 \mathrm{a} R^{*}, 3 S^{*}, 5 S^{*}, 7 \mathrm{a} R^{*}, 7 \mathrm{~b} R^{*}, 8 R^{*}, 9 R^{*}, 9 \mathrm{a} R^{*}\right)$-5-hydroxy-9a-isopropoxy-1,1,8-trimethyl-9(tetradecanoyloxy)-1,1a,3,5,6,7,7a,8,9,9a-decahydro-3,7b-epoxycyclopropa[3,4]benzo[1,2-e]azulene-4carboxylate (32)

The procedure was modified from the Yamaguchi esterification described by Santa Lucia et.al. ${ }^{9}$ Alcohol 31 $(20 \mathrm{mg}, 38 \mu \mathrm{mol})$ was dissolved in anhydrous THF $(0.5 \mathrm{~mL})$ and to this was added benzoyl chloride $(20 \mu \mathrm{L}$, $0.17 \mathrm{mmol}$ ), myristic acid (39 $\mathrm{mg}, 0.17 \mathrm{mmol})$, triethylamine $(40 \mu \mathrm{L}, 0.29 \mathrm{mmol}$ ) and DMAP (8.9 $\mathrm{mg}, 73$ $\mu \mathrm{mol})$ under an argon atmosphere. The reaction mixture was then stirred for $2 \mathrm{~d}$, followed by workup through partitioning between a saturated $\mathrm{NaHCO}_{3}$ solution and $15 \%$ ethyl acetate in petroleum spirit ( $3 \mathrm{x}$ $1.5 \mathrm{~mL}$ ). The combined organic extracts were dried over anhydrous $\mathrm{Na}_{2} \mathrm{SO}_{4}$ and concentrated in vacuo. The crude residue was purified via chromatography with neutral alumina ( $4 \%$ ethyl acetate in petroleum spirit) 
to afford the TBS-myristate as a colourless oil $(22 \mathrm{mg})$, which was then treated with methanol $(4 \mathrm{~mL})$ and a catalytic amount of Amberlyst I15 (5 mg) for $7 \mathrm{~d}$. After filtration and concentration, the crude was purified via chromatography with neutral alumina ( $20 \%$ ethyl acetate in petroleum spirit) to give the desired hydroxy-myristate 32 as a clear oil (10 mg, 40\% over 2 steps [ $63 \%$ brsm over 2 steps]). Note: The unreacted TBSO-myristate $(8.6 \mathrm{mg}, 39 \%$ ) was recovered from the silyl deprotection (Step 2).

${ }^{1} \mathrm{H}$ NMR $\left(500 \mathrm{MHz}, \mathrm{C}_{6} \mathrm{D}_{6}\right) \delta 0.86(\mathrm{t}, J=7.2 \mathrm{~Hz}, 3 \mathrm{H}), 0.92(\mathrm{t}, J=7.0 \mathrm{~Hz}, 3 \mathrm{H}), 0.96(\mathrm{~d}, J=6.2 \mathrm{~Hz}, 3 \mathrm{H}), 1.24-1.35$ $(\mathrm{m}, 33 \mathrm{H}), 1.43-1.52(\mathrm{~m}, 1 \mathrm{H}), 1.52-1.65(\mathrm{~m}, 2 \mathrm{H}), 1.68$ (quin, J=7.2 Hz, $2 \mathrm{H}), 2.03$ (dd, J=13.0, $6.4 \mathrm{~Hz}, 1 \mathrm{H}$ ), $2.34(\mathrm{td}, J=7.4,1.7 \mathrm{~Hz}, 2 \mathrm{H}$ ), 2.47 (dq, J=12.1, $7.0 \mathrm{~Hz}, 1 \mathrm{H}$ ), 2.75 (dd, J=13.2, $6.2 \mathrm{~Hz}, 1 \mathrm{H}$ ), 3.78 (spt, J=6.1 Hz, 1 H), 3.87 (qd, J=7.0, $0.7 \mathrm{~Hz}, 2 \mathrm{H}), 4.49(\mathrm{~d}, J=12.5 \mathrm{~Hz}, 1 \mathrm{H}), 4.99$ (t, J=1.8 Hz, $1 \mathrm{H}), 5.10$ (dd, J=7.3, $1.1 \mathrm{~Hz}, 1 \mathrm{H}$ ), $5.20(\mathrm{~d}, J=1.8 \mathrm{~Hz}, 1 \mathrm{H}), 6.07(\mathrm{t}, J=2.4 \mathrm{~Hz}, 1 \mathrm{H})$.

${ }^{13} \mathrm{C}$ NMR $\left(176 \mathrm{MHz}, \mathrm{C}_{6} \mathrm{D}_{6}\right) \delta 11.1,14.3,14.7,18.5,22.7,23.5,24.4,24.6,25.6,25.7,26.1,28.0,29.9,30.1$, $30.2,30.3,30.42,30.47,30.48,30.51,32.7,33.7,34.8,40.2,47.7,61.3,68.0,72.07,72.13,72.8,76.8,89.4$, $129.6,136.7,139.0,163.0,166.6,172.4$.

HRMS (El+) calculated for $\mathrm{C}_{38} \mathrm{H}_{60} \mathrm{NaO}_{7}: 651.4237$; found: 651.4231 .

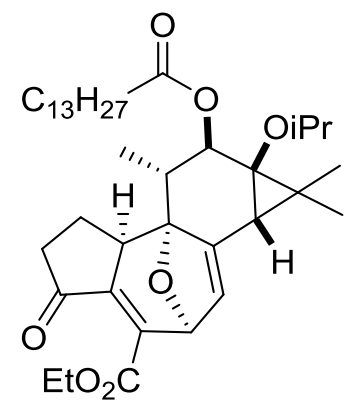

Ethyl $\quad\left(1 \mathrm{a} R^{*}, 3 S^{*}, 7 \mathrm{a} S^{*}, 7 \mathrm{~b} R^{*}, 8 R^{*}, 9 R^{*}, 9 \mathrm{a} R^{*}\right)-9 \mathrm{a}$-isopropoxy-1,1,8-trimethyl-5-oxo-9-(tetradecanoyloxy)1,1a,3,5,6,7,7a,8,9,9a-decahydro-3,7b-epoxycyclopropa[3,4]benzo[1,2-e]azulene-4-carboxylate (33)

The hydroxy-myristate 32 (10 mg, $16 \mu \mathrm{mol}$ ) was treated with Dess-Martin periodinane (11 mg, $26 \mu \mathrm{mol}$ ) and $\mathrm{NaHCO}_{3}(4.0 \mathrm{mg}, 48 \mu \mathrm{mol})$ in anhydrous DCM. The reaction was stirred for $20 \mathrm{~h}$ at which point TLC indicated no sign of starting material. The reaction mixture was then partitioned with a saturated $\mathrm{NaHCO}_{3}$ solution and DCM $(3 \times 1 \mathrm{~mL})$, and the combined organic extracts dried over anhydrous $\mathrm{Na}_{2} \mathrm{SO}_{4}$ and concentrated in vacuo. The desired keto-myristate $\mathbf{3 3}$ was obtained as a clear liquid (10 $\mathrm{mg}$, quantitative) after chromatography with neutral alumina ( $20 \%$ ethyl acetate in petroleum spirit).

${ }^{1} \mathrm{H}$ NMR (500 MHz, $\left.C_{6} \mathrm{D}_{6}\right) \delta 0.73(\mathrm{qd}, J=12.0,7.9 \mathrm{~Hz}, 1 \mathrm{H}), 0.92(\mathrm{t}, J=7.0 \mathrm{~Hz}, 3 \mathrm{H}), 0.96(\mathrm{~d}, J=6.2 \mathrm{~Hz}, 3 \mathrm{H}), 1.08$ (d, J=2.2 Hz, $1 \mathrm{H}), 1.16(\mathrm{t}, J=7.2 \mathrm{~Hz}, 3 \mathrm{H}), 1.21(\mathrm{~s}, 3 \mathrm{H}), 1.24-1.33(\mathrm{~m}, 28 \mathrm{H}), 1.38(\mathrm{dt}, J=11.4,7.7 \mathrm{~Hz}, 1 \mathrm{H})$, 1.69 (s, $3 \mathrm{H}$ ), $1.64-1.74(\mathrm{~m}, 1 \mathrm{H}), 1.90$ (dd, J=17.6, $7.7 \mathrm{~Hz}, 1 \mathrm{H}$ ), 2.30 (dt, J=12.5, 6.6 Hz, $1 \mathrm{H}$ ), 2.34 (td, J=7.3, $1.5 \mathrm{~Hz}, 2 \mathrm{H}$ ), $2.72(\mathrm{dd}, J=12.8,7.0 \mathrm{~Hz}, 1 \mathrm{H}), 3.63(\mathrm{spt}, J=6.2 \mathrm{~Hz}, 1 \mathrm{H}), 4.23(\mathrm{dq}, J=10.6,7.0 \mathrm{~Hz}, 1 \mathrm{H}), 4.25$ (dq, $J=10.6,7.0 \mathrm{~Hz}, 1 \mathrm{H}), 4.40(\mathrm{~d}, J=12.1 \mathrm{~Hz}, 1 \mathrm{H}), 4.79(\mathrm{dt}, J=2.2,1.1 \mathrm{~Hz}, 1 \mathrm{H}), 6.10(\mathrm{t}, J=2.4 \mathrm{~Hz}, 1 \mathrm{H})$.

${ }^{13} \mathrm{C}$ NMR $\left(125 \mathrm{MHz}, \mathrm{C}_{6} \mathrm{D}_{6}\right) \delta 11.1,14.5,14.7,18.4,22.4,22.7,23.5,24.4,24.6,25.7,25.9,28.2,29.9,30.1$, $30.2,30.3,30.4,30.48$ (2C), 30.51, 32.7, 34.8, 38.3, 39.7, 41.3, 61.8, 67.9, 71.9, 72.7, 78.0, 89.9, 135.8, $137.6,139.2,140.2,166.5,172.4,201.7$.

HRMS (EI+) calculated for $\mathrm{C}_{38} \mathrm{H}_{58} \mathrm{NaO}_{7}: 649.4080$; found: 649.4075 . 


\section{References}

1 Stevens, R. V., Angle, S. R., Kloc, K., Mak, K. F., Trueblood, K. N., Liu, Y.-X. J. Org. Chem., 1986, $51,4347-4353$.

2 Lee, C.-L., Huang, C.-H., Wang, H.-C., Chuang, d.-W., Wu, M.-J., Wang, S.-Y., Hwang, T.-L., Wu, C.-C., Chen, Y.-L., Chang, f.-R., Wu, Y.-C. Org. Biomol. Chem. 2011, 9, $70-73$.

3 Saa, J. M., Llobera, A., Deya, P. M. Chem. Lett. 1987, $771-774$.

4 Wurm, G.; Gurka, H.-J.; Geres, U. Arch. Pharm., 1986, $1106-1113$.

5 Edwards, M. G., Paxton, R. J., Pugh, D. S., Whitwood, A. C., Taylor, R. J. K. Synthesis, 2008, 3279 - 3288; Edwards, M. G., Paxton, R. J., Pugh, D. S., Taylor, R. J. K. Synlett, 2007, 521 - 524; unpublished notes from Prof R. J. K. Taylor.

6 Taber, D. F., DeMatteo, P. W., Hassan, R. A. Org. Synth., 2013, 90, $350-357$.

Aponick, A., Li, C.-Y., Malinge, J., Marques, E. F. Org. Lett., 2009, 11, $4624-4627$.

8 Krainz, T., Chow, S., Korica, N., Bernhardt, P. V., Boyle, G. M., Parsons, P. G., Davies, H. M. L., Williams, C. M. Eur. J. Org. Chem., 2016, $41-44$.

9 Dhimitruka, I.; SantaLucia Jr., J. Org. Lett., 2006, 8, 47 - 50. 

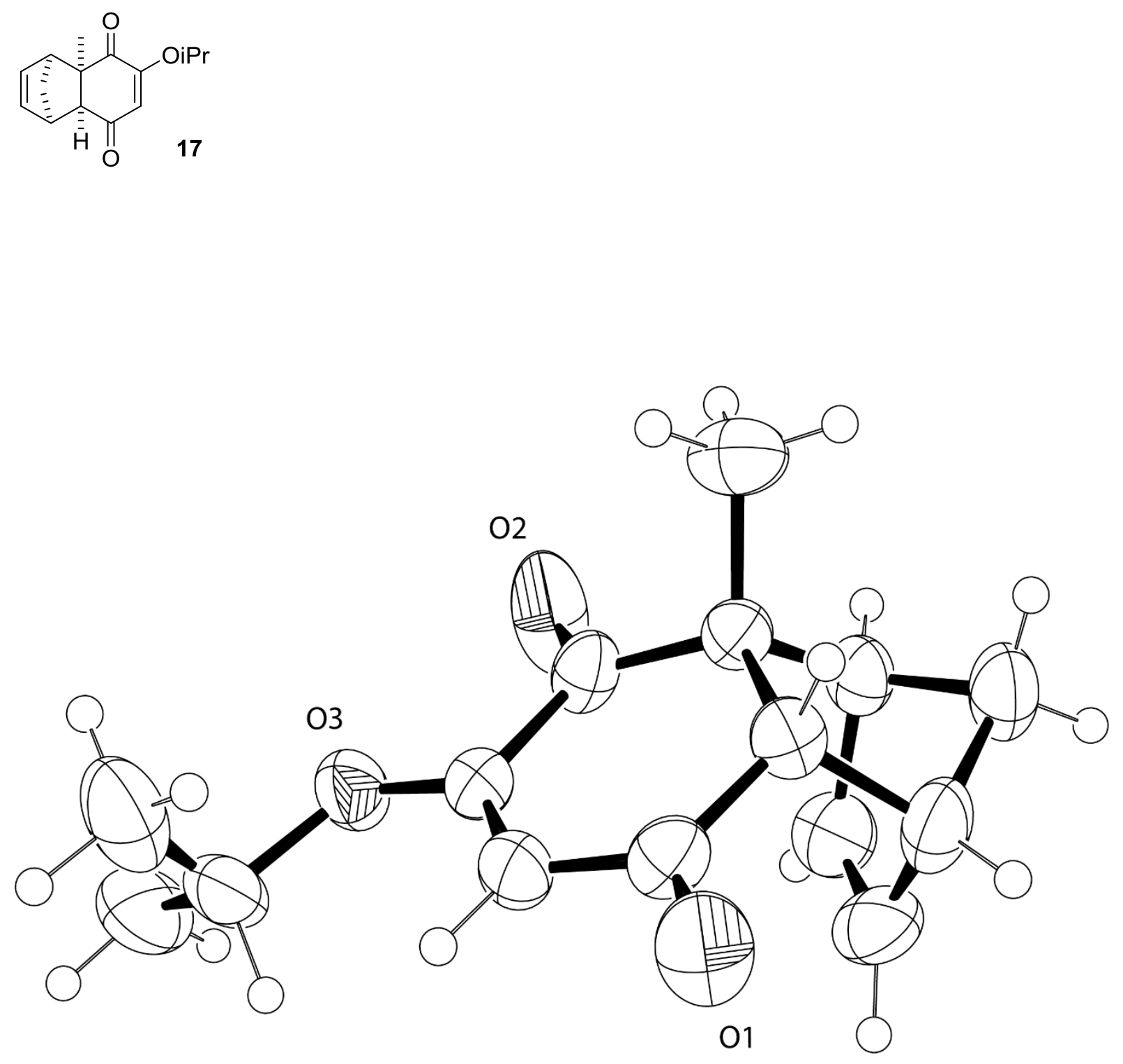
Identification code

Empirical formula

Formula weight

Temperature

Wavelength

Crystal system

Space group

Unit cell dimensions

Volume

Z

Density (calculated)

Absorption coefficient

$\mathrm{F}(000)$

Crystal size

Theta range for data collection

Index ranges

Reflections collected

Independent reflections

Completeness to theta $=24.999^{\circ}$

Absorption correction

Max. and min. transmission

Refinement method

Data / restraints / parameters

Goodness-of-fit on $\mathrm{F}^{2}$

Final $R$ indices [ $>2$ sigma(I)]

$\mathrm{R}$ indices (all data)

Extinction coefficient

Largest diff. peak and hole 860tk3 (CCDC 1955317)

$\mathrm{C}_{15} \mathrm{H}_{18} \mathrm{O}_{3}$

246.29

190(2) K

$0.7107 \AA$

Monoclinic

$P 2{ }_{1} / c$

$a=6.365(2) \AA \quad \alpha=90^{\circ}$.

$b=21.464(5) \AA \quad \beta=100.69(3)^{\circ}$.

c $=9.837(3) \AA \quad \gamma=90^{\circ}$.

$1320.6(7) \AA^{3}$

4

$1.239 \mathrm{Mg} / \mathrm{m}^{3}$

$0.085 \mathrm{~mm}^{-1}$

528

$0.600 \times 0.600 \times 0.400 \mathrm{~mm}^{3}$

3.257 to $24.999^{\circ}$.

$-6<=\mathrm{h}<=7,-25<=\mathrm{k}<=24,-11<=1<=11$

9703

$2329[R($ int $)=0.0260]$

$99.9 \%$

Semi-empirical from equivalents

1 and 0.969

Full-matrix least-squares on $\mathrm{F}^{2}$

2329 / 0 / 166

1.043

$R 1=0.0480, w R 2=0.1090$

$\mathrm{R} 1=0.0603, \mathrm{wR} 2=0.1158$

$\mathrm{n} / \mathrm{a}$

0.171 and -0.146 e. $\AA^{-3}$ 

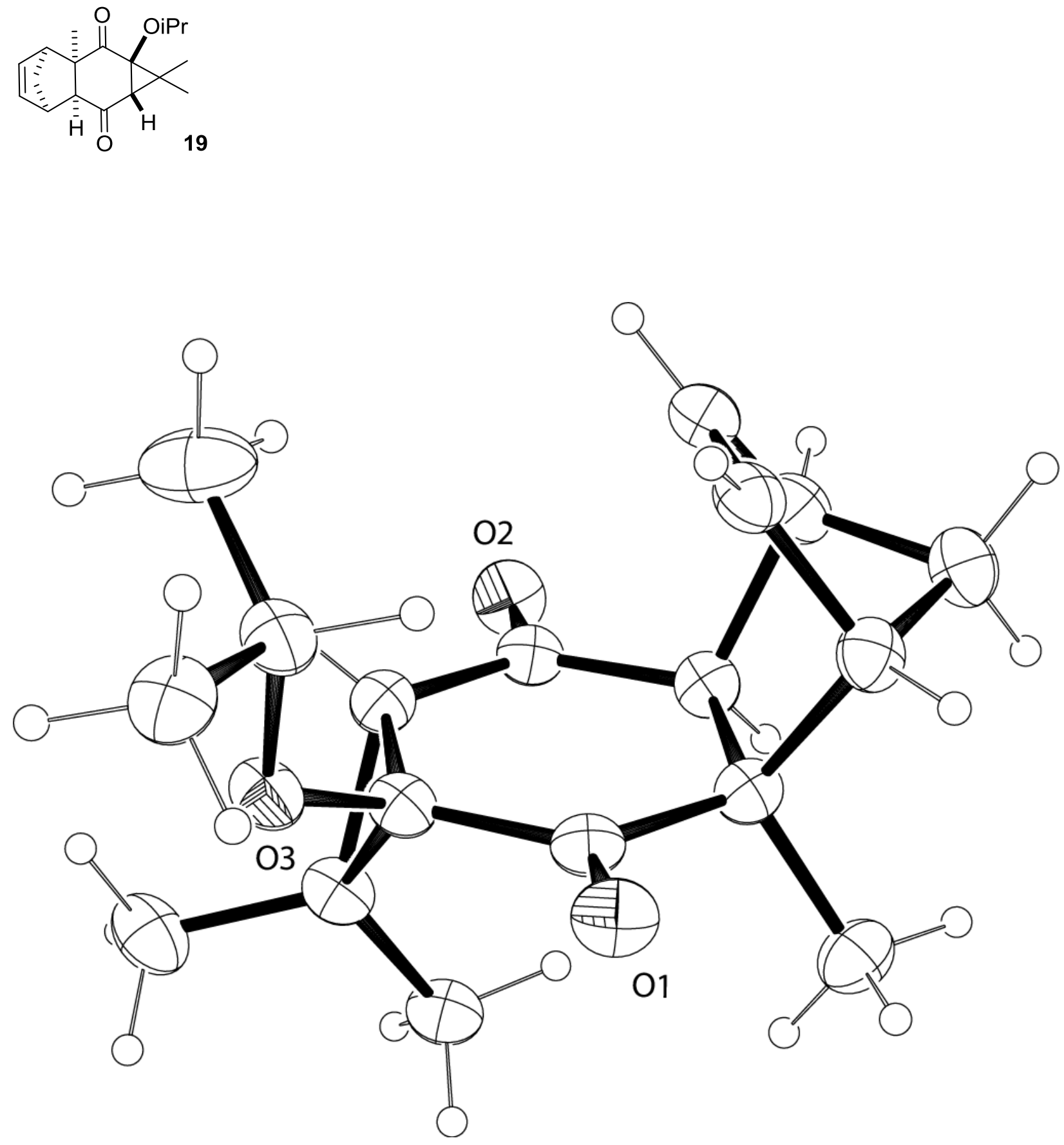
Identification code

Empirical formula

Formula weight

Temperature

Wavelength

Crystal system

Space group

Unit cell dimensions

Volume

Z

Density (calculated)

Absorption coefficient

$\mathrm{F}(000)$

Crystal size

Theta range for data collection

Index ranges

Reflections collected

Independent reflections

Completeness to theta $=62.478^{\circ}$

Absorption correction

Max. and min. transmission

Refinement method

Data / restraints / parameters

Goodness-of-fit on $\mathrm{F}^{2}$

Final $R$ indices [ $>2$ sigma(I)]

$\mathrm{R}$ indices (all data)

Extinction coefficient

Largest diff. peak and hole 1112tk-111-75 (CCDC 1955318)

$\mathrm{C}_{18} \mathrm{H}_{24} \mathrm{O}_{3}$

288.37

190(2) K

$1.5418 \AA$

Monoclinic

$P 2_{1} / n$

$a=8.3706(8) \AA \quad \alpha=90^{\circ}$.

$b=17.0190(15) \AA \quad \beta=99.617(8)^{\circ}$.

$\mathrm{c}=11.1609(9) \AA \quad \gamma=90^{\circ}$.

$1567.6(2) \AA^{3}$

4

$1.222 \mathrm{Mg} / \mathrm{m}^{3}$

$0.650 \mathrm{~mm}^{-1}$

624

$0.2 \times 0.2 \times 0.2 \mathrm{~mm}^{3}$

4.785 to $62.478^{\circ}$.

$-9<=\mathrm{h}<=9,-19<=\mathrm{k}<=11,-10<=\mid<=12$

6198

$2484[R($ int $)=0.0393]$

$99.1 \%$

Semi-empirical from equivalents

1 and 0.605

Full-matrix least-squares on $\mathrm{F}^{2}$

2484 / 0 / 195

1.076

$\mathrm{R} 1=0.0631, \mathrm{wR} 2=0.1548$

$\mathrm{R} 1=0.0759, \mathrm{wR} 2=0.1676$

$\mathrm{n} / \mathrm{a}$

0.393 and -0.226 e. $\AA^{-3}$ 

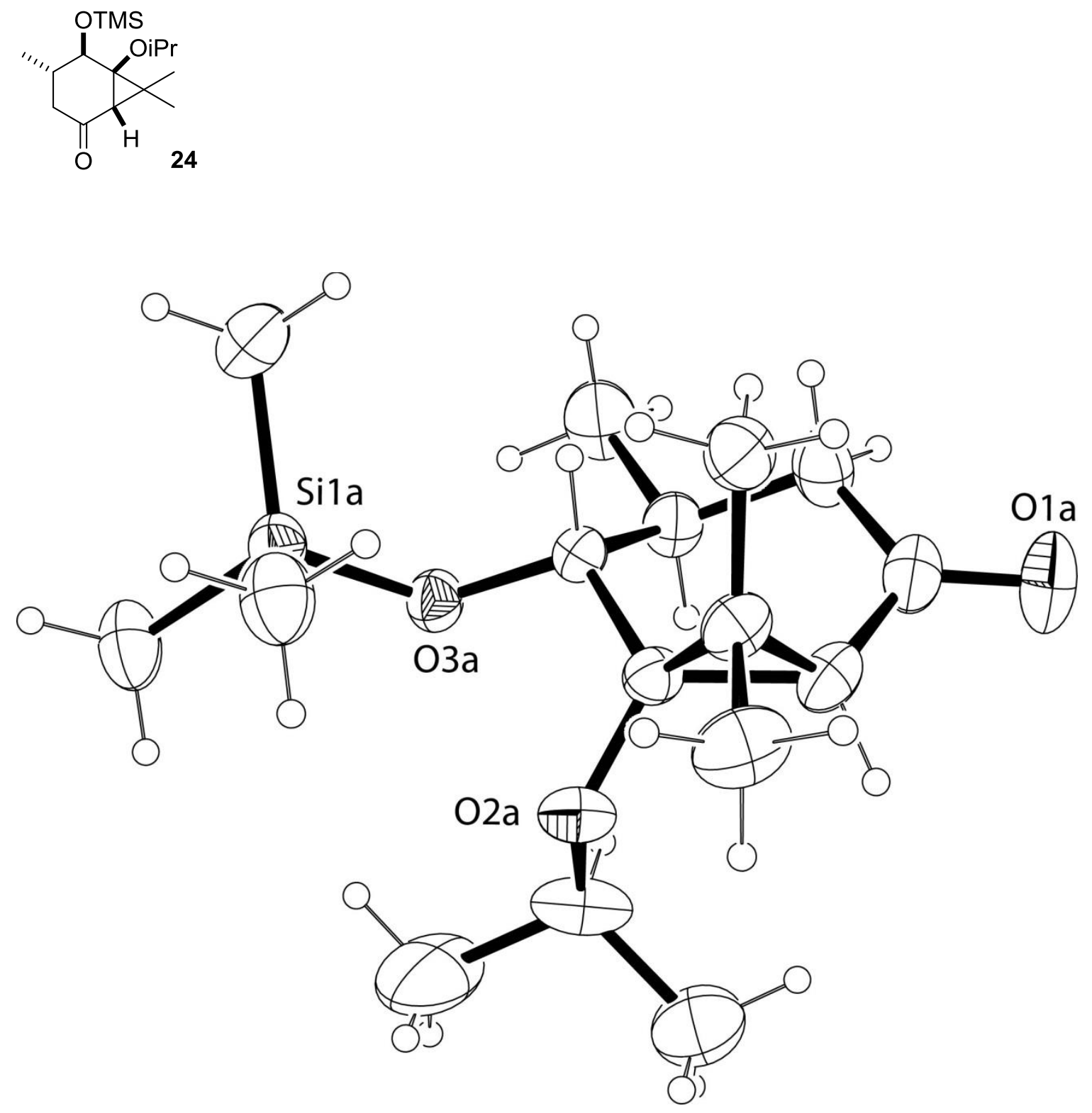
Identification code

Empirical formula

Formula weight

Temperature

Wavelength

Crystal system

Space group

Unit cell dimensions

Volume

Z

Density (calculated)

Absorption coefficient

$\mathrm{F}(000)$

Crystal size

Theta range for data collection

Index ranges

Reflections collected

Independent reflections

Completeness to theta $=25.00^{\circ}$

Absorption correction

Max. and min. transmission

Refinement method

Data / restraints / parameters

Goodness-of-fit on $\mathrm{F}^{2}$

Final $R$ indices [ $I>2$ sigma(I)]

$\mathrm{R}$ indices (all data)

Largest diff. peak and hole 1096sc1 (CCDC 1955319)

$\mathrm{C}_{16} \mathrm{H}_{30} \mathrm{O}_{3} \mathrm{Si}$

298.49

190(2) K

$0.71073 \AA$

Triclinic

$P \overline{1}$

$\mathrm{a}=10.034(3) \AA \quad \alpha=80.65(2)^{\circ}$.

$\mathrm{b}=12.968(3) \AA \quad \beta=88.39(2)^{\circ}$.

$\mathrm{c}=14.934(3) \AA \quad \gamma=84.63(2)^{\circ}$.

1908.9(8) $\AA^{3}$

4

$1.039 \mathrm{Mg} / \mathrm{m}^{3}$

$0.128 \mathrm{~mm}^{-1}$

656

$0.4 \times 0.2 \times 0.1 \mathrm{~mm}^{3}$

2.97 to $29.42^{\circ}$.

$-13<=\mathrm{h}<=12,-17<=\mathrm{k}<=17,-20<=\mathrm{l}<=12$

19545

$9071[R($ int $)=0.0499]$

$99.8 \%$

Semi-empirical from equivalents

1 and 0.38943

Full-matrix least-squares on $\mathrm{F}^{2}$

9071 / 0 / 365

1.021

$R 1=0.0746, w R 2=0.1711$

$R 1=0.1323, w R 2=0.2068$

0.286 and -0.216 e. $\AA^{-3}$ 

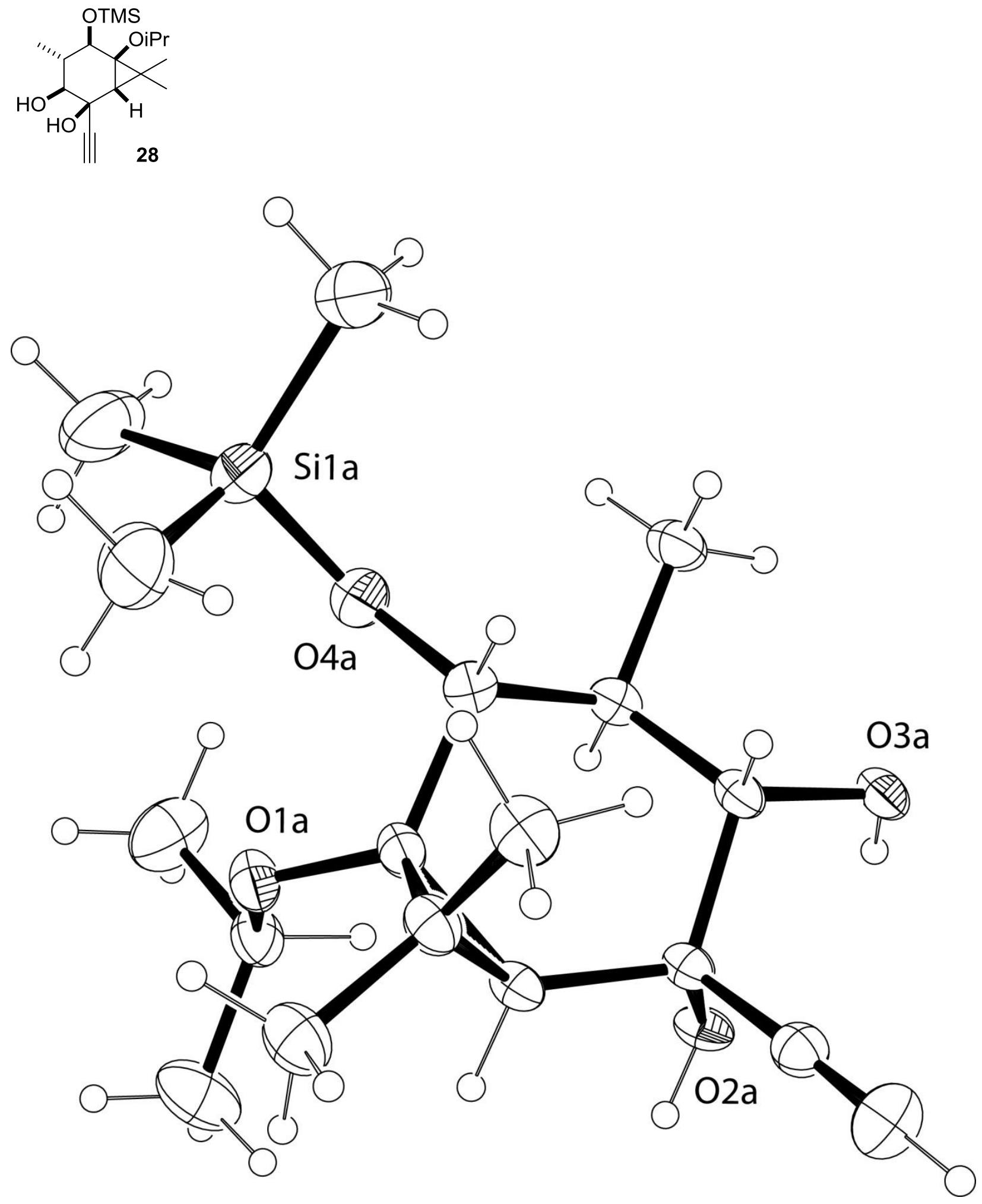
Identification code

Empirical formula

Formula weight

Temperature

Wavelength

Crystal system

Space group

Unit cell dimensions

Volume

Z

Density (calculated)

Absorption coefficient

$\mathrm{F}(000)$

Crystal size

Theta range for data collection

Index ranges

Reflections collected

Independent reflections

Completeness to theta $=62.50^{\circ}$

Absorption correction

Max. and min. transmission

Refinement method

Data / restraints / parameters

Goodness-of-fit on $\mathrm{F}^{2}$

Final $R$ indices [I>2sigma(I)]

$\mathrm{R}$ indices (all data)

Largest diff. peak and hole 1323sc2 (CCDC 1955320)

$\mathrm{C}_{18} \mathrm{H}_{32} \mathrm{O}_{4} \mathrm{Si}$

340.53

190(2) K

$1.54184 \AA$

Triclinic

$P \overline{1}$

$a=10.3455(10) \AA \quad \alpha=73.468(7)^{\circ}$.

$b=13.8560(13) \AA \quad \beta=86.752(7)^{\circ}$.

$c=15.6403(12) \AA \quad \gamma=74.998(8)^{\circ}$.

2075.7(3) $\AA^{3}$

4

$1.090 \mathrm{Mg} / \mathrm{m}^{3}$

$1.123 \mathrm{~mm}^{-1}$

744

$0.3 \times 0.1 \times 0.04 \mathrm{~mm}^{3}$

3.44 to $62.50^{\circ}$.

$-11<=\mathrm{h}<=10,-12<=\mathrm{k}<=15,-17<=\mathrm{l}<=17$

16171

$6526[R($ int $)=0.0539]$

$99.0 \%$

Semi-empirical from equivalents

1 and 0.87037

Full-matrix least-squares on $\mathrm{F}^{2}$

6526 / 0 / 419

1.029

$R 1=0.0711, w R 2=0.2017$

$\mathrm{R} 1=0.0917, w R 2=0.2370$

1.012 and -0.483 e. $\AA^{-3}$ 

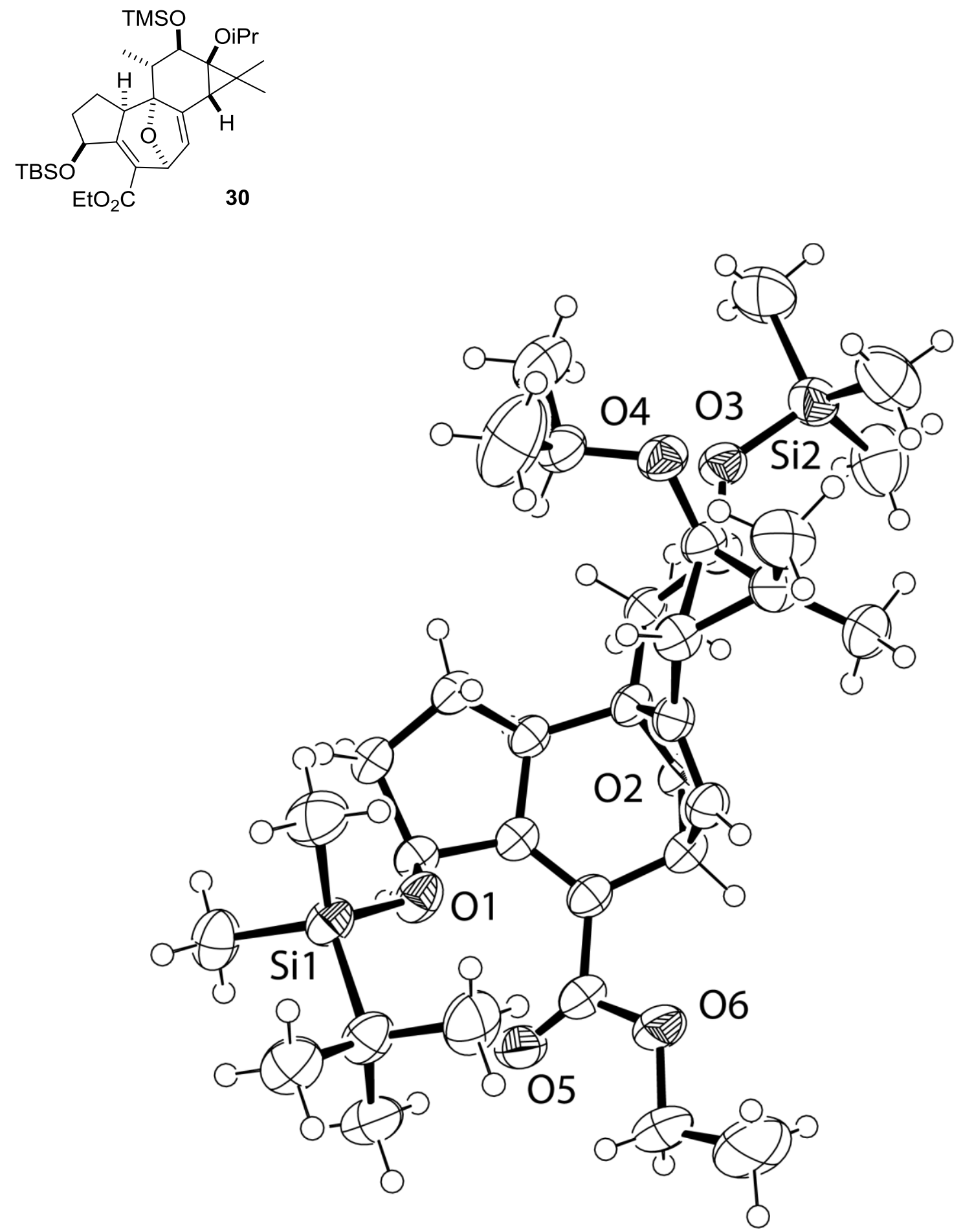
Identification code

Empirical formula

Formula weight

Temperature

Wavelength

Crystal system

Space group

Unit cell dimensions

Volume

Z

Density (calculated)

Absorption coefficient

$\mathrm{F}(000)$

Crystal size

Theta range for data collection

Index ranges

Reflections collected

Independent reflections

Completeness to theta $=62.496^{\circ}$

Absorption correction

Max. and min. transmission

Refinement method

Data / restraints / parameters

Goodness-of-fit on $\mathrm{F}^{2}$

Final $R$ indices [ $>2$ sigma(I)]

$\mathrm{R}$ indices (all data)

Extinction coefficient

Largest diff. peak and hole 1207sc1 (CCDC 1955321)

$\mathrm{C}_{33} \mathrm{H}_{56} \mathrm{O}_{6} \mathrm{Si}_{2}$

604.95

190(2) K

$1.5418 \AA$

Monoclinic

$P 2_{1} / c$

$a=13.2671(6) \AA$

$\alpha=90^{\circ}$.

$\mathrm{b}=13.5663(8) \AA$

$\beta=93.323(4)^{\circ}$.

C $=20.4372(13) \AA$

$\gamma=90^{\circ}$.

3672.2(4) $\AA^{3}$

4

$1.094 \mathrm{Mg} / \mathrm{m}^{3}$

$1.173 \mathrm{~mm}^{-1}$

1320

$0.5 \times 0.5 \times 0.2 \mathrm{~mm}^{3}$

3.337 to $62.496^{\circ}$.

$-10<=\mathrm{h}<=15,-15<=\mathrm{k}<=14,-22<=\mathrm{k}<=23$

17861

$5727[R($ int $)=0.0644]$

$97.5 \%$

Semi-empirical from equivalents

1 and 0.47

Full-matrix least-squares on $\mathrm{F}^{2}$

5727 / 0 / 384

1.067

$R 1=0.0599, w R 2=0.1715$

$\mathrm{R} 1=0.0666, \mathrm{wR} 2=0.1843$

$\mathrm{n} / \mathrm{a}$

0.452 and -0.409 e. $\AA^{-3}$ 


\section{NMR spectra}

scC109.001.001.1r.esp
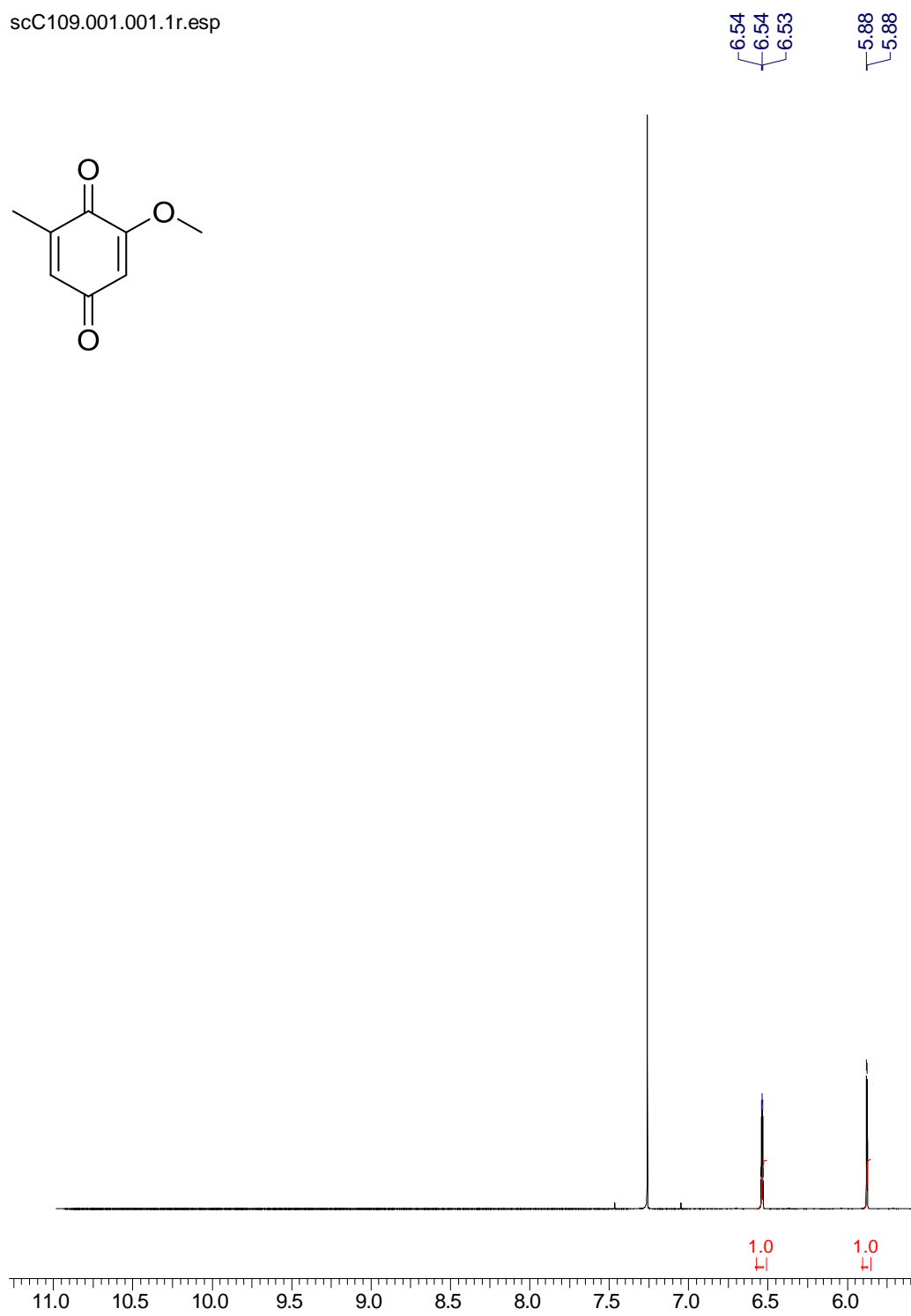

$500 \mathrm{MHz}{ }^{1} \mathrm{H}$ NMR spectrum of $15\left(\mathrm{CDCl}_{3}\right)$. 
<smiles>COC1=CC(=O)C=C(C)C1=O</smiles>
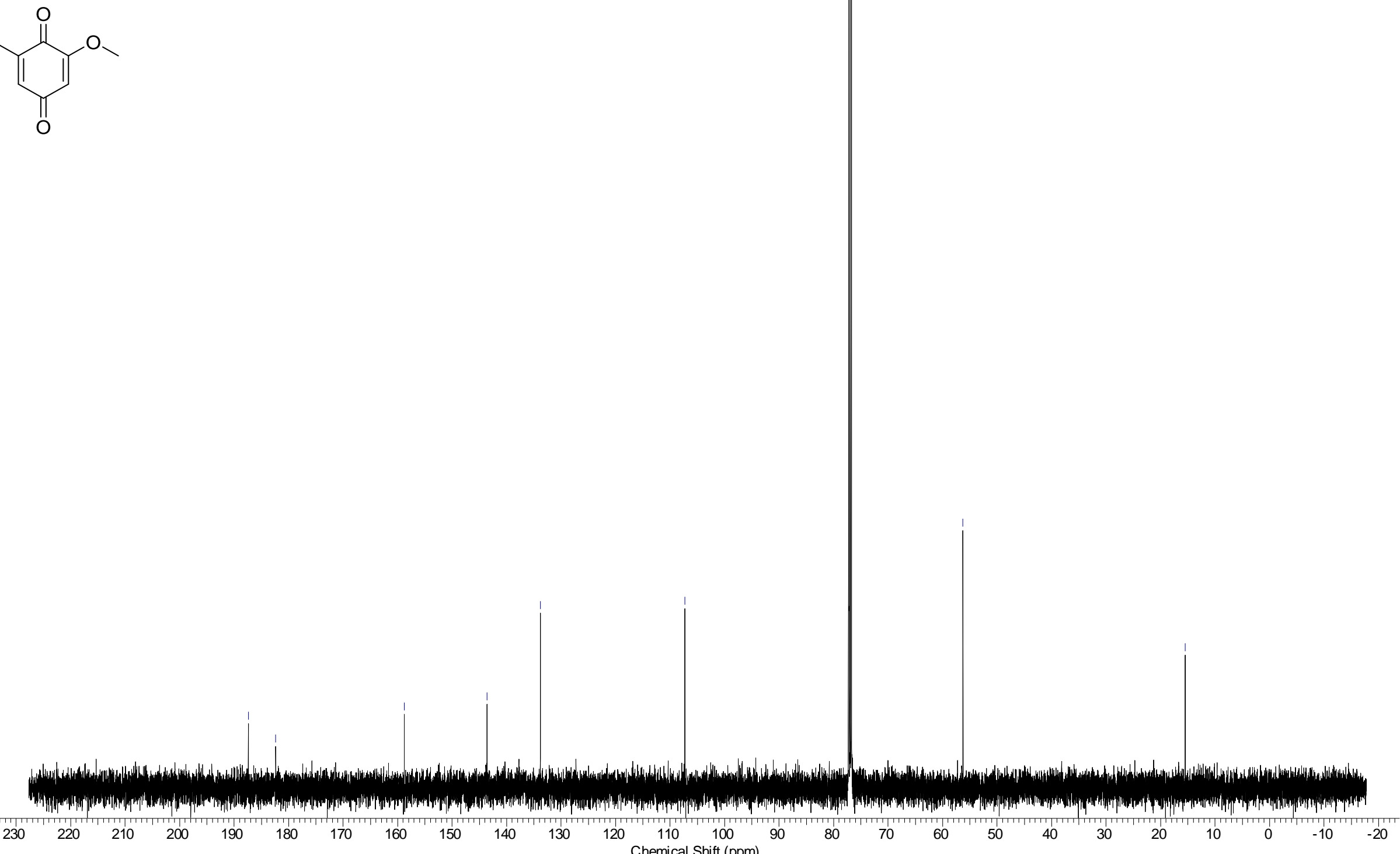

$125 \mathrm{MHz}{ }^{13} \mathrm{C}$ NMR spectrum of $15\left(\mathrm{CDCl}_{3}\right)$. 

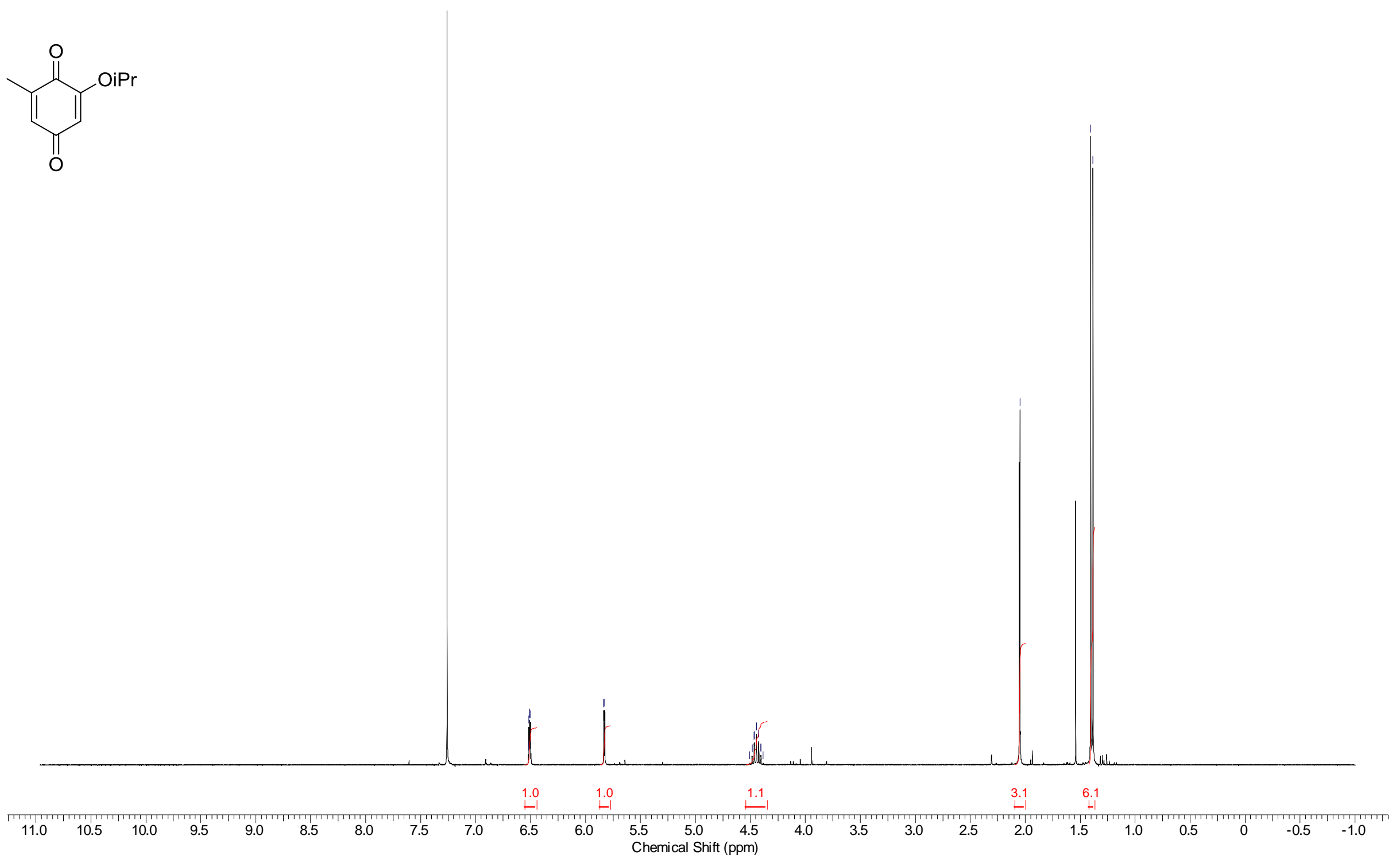

$300 \mathrm{MHz}{ }^{1} \mathrm{H}$ NMR spectrum of $16\left(\mathrm{CDCl}_{3}\right)$. 

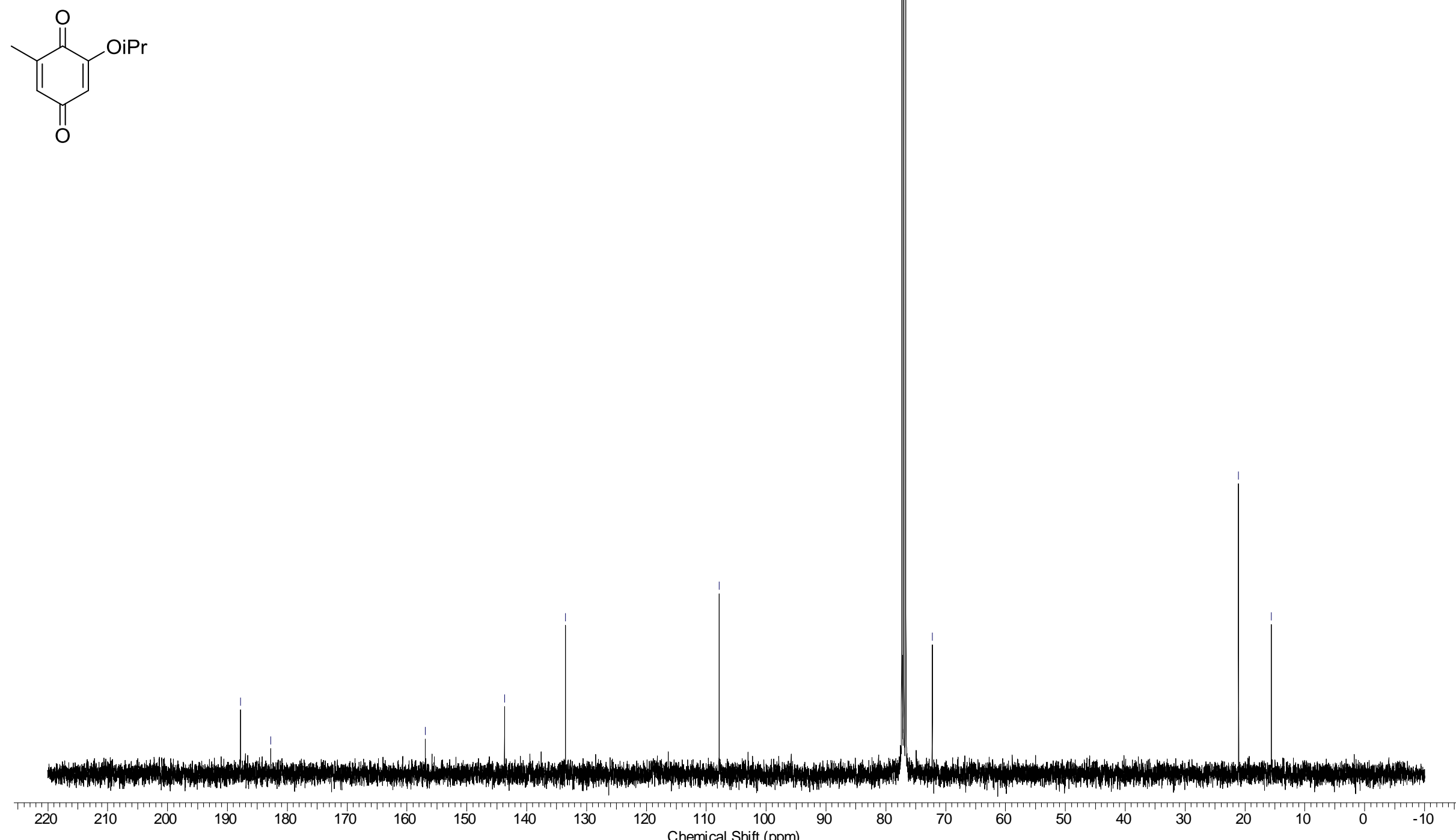

$100 \mathrm{MHz}{ }^{13} \mathrm{C}$ NMR spectrum of $16\left(\mathrm{CDCl}_{3}\right)$. 

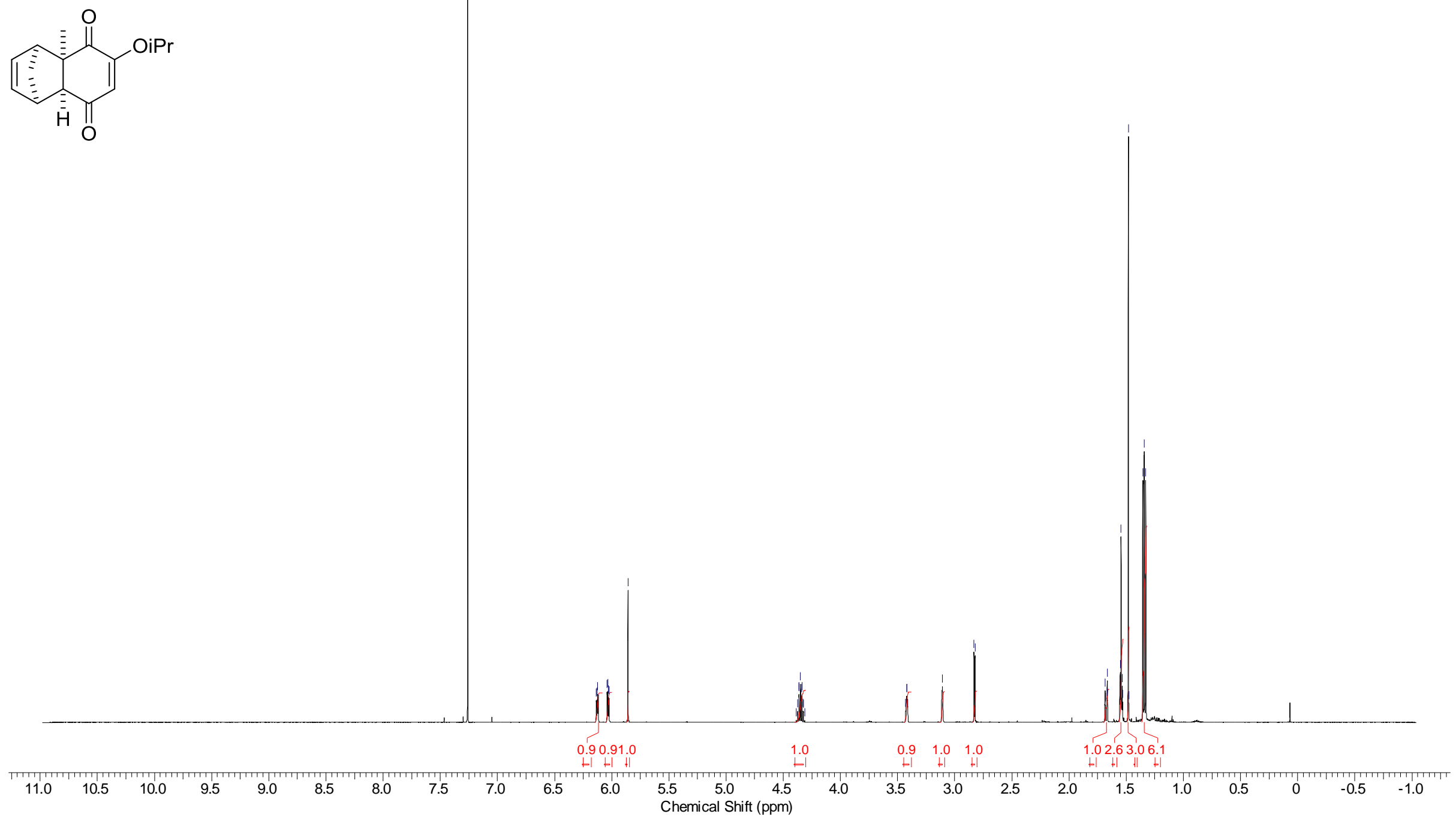

$400 \mathrm{MHz}{ }^{1} \mathrm{H}$ NMR spectrum of $17\left(\mathrm{CDCl}_{3}\right)$. 


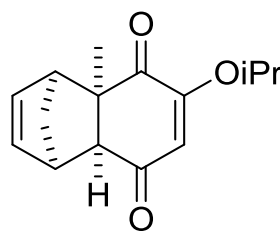

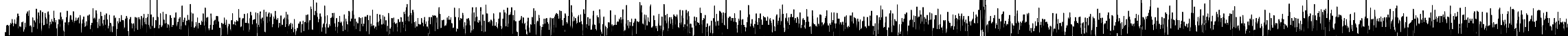

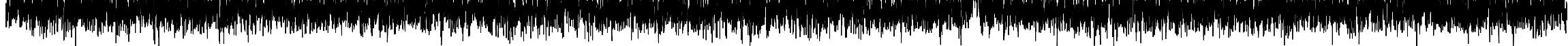

$100 \mathrm{MHz}{ }^{13} \mathrm{C}$ NMR spectrum of $17\left(\mathrm{CDCl}_{3}\right)$. 


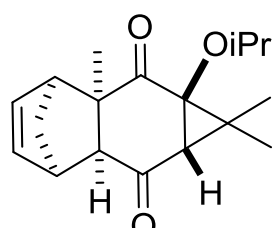

$\mathrm{H}$

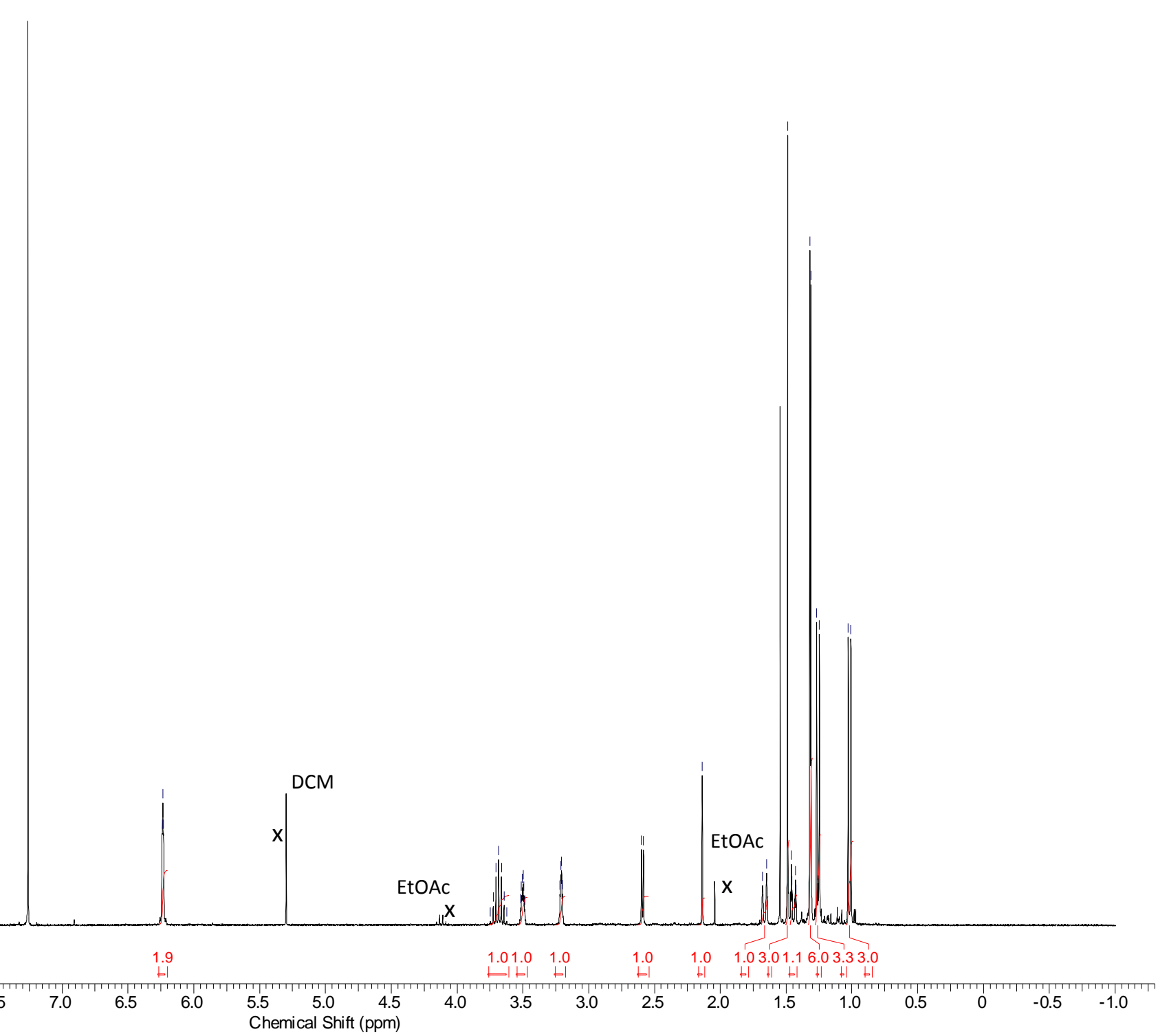

$400 \mathrm{MHz}{ }^{1} \mathrm{H}$ NMR spectrum of $19\left(\mathrm{CDCl}_{3}\right)$. 

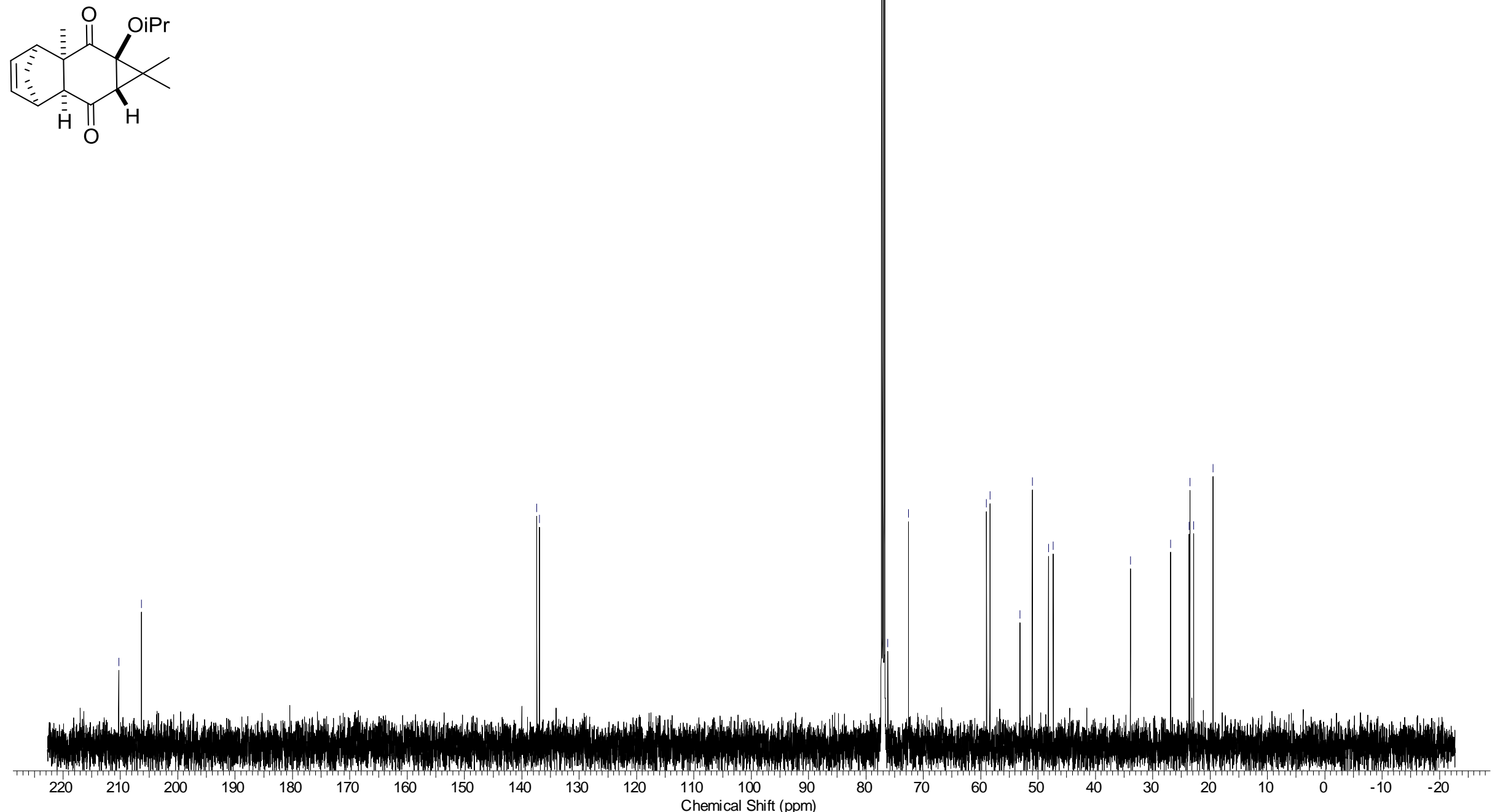

$125 \mathrm{MHz}{ }^{13} \mathrm{C}$ NMR spectrum of $19\left(\mathrm{CDCl}_{3}\right)$. 

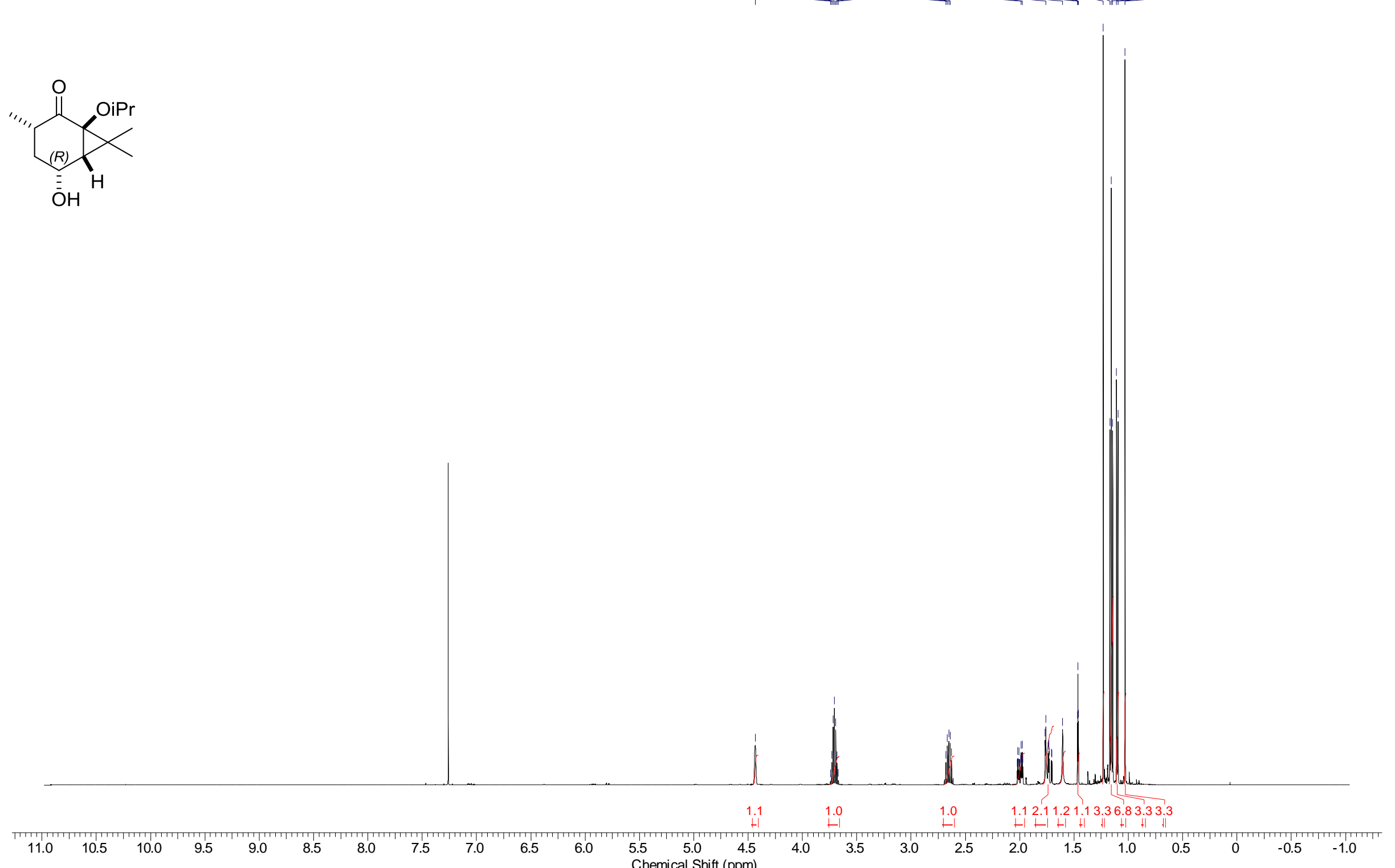

$500 \mathrm{MHz}{ }^{1} \mathrm{H}$ NMR spectrum of $\left(5 R^{*}\right)-22\left(\mathrm{CDCl}_{3}\right)$. 
(R)

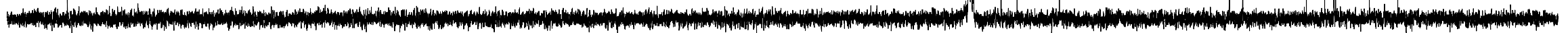

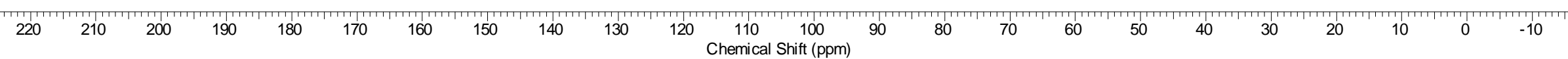

$100 \mathrm{MHz}{ }^{13} \mathrm{C}$ NMR spectrum of $\left(5 R^{*}\right)-22\left(\mathrm{CDCl}_{3}\right)$. 

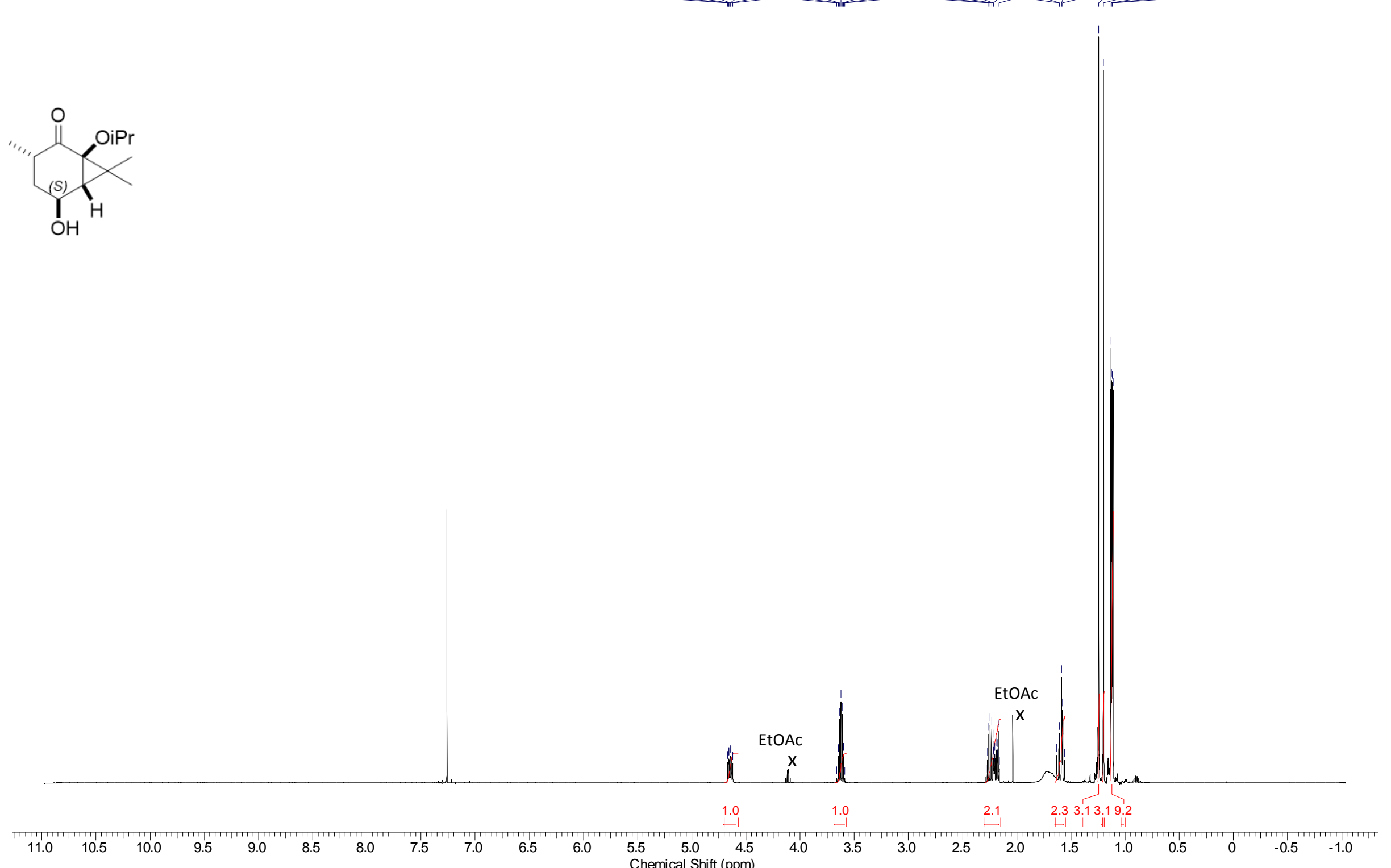

$500 \mathrm{MHz}{ }^{1} \mathrm{H}$ NMR spectrum of $\left(5 S^{*}\right)-22\left(\mathrm{CDCl}_{3}\right)$. 

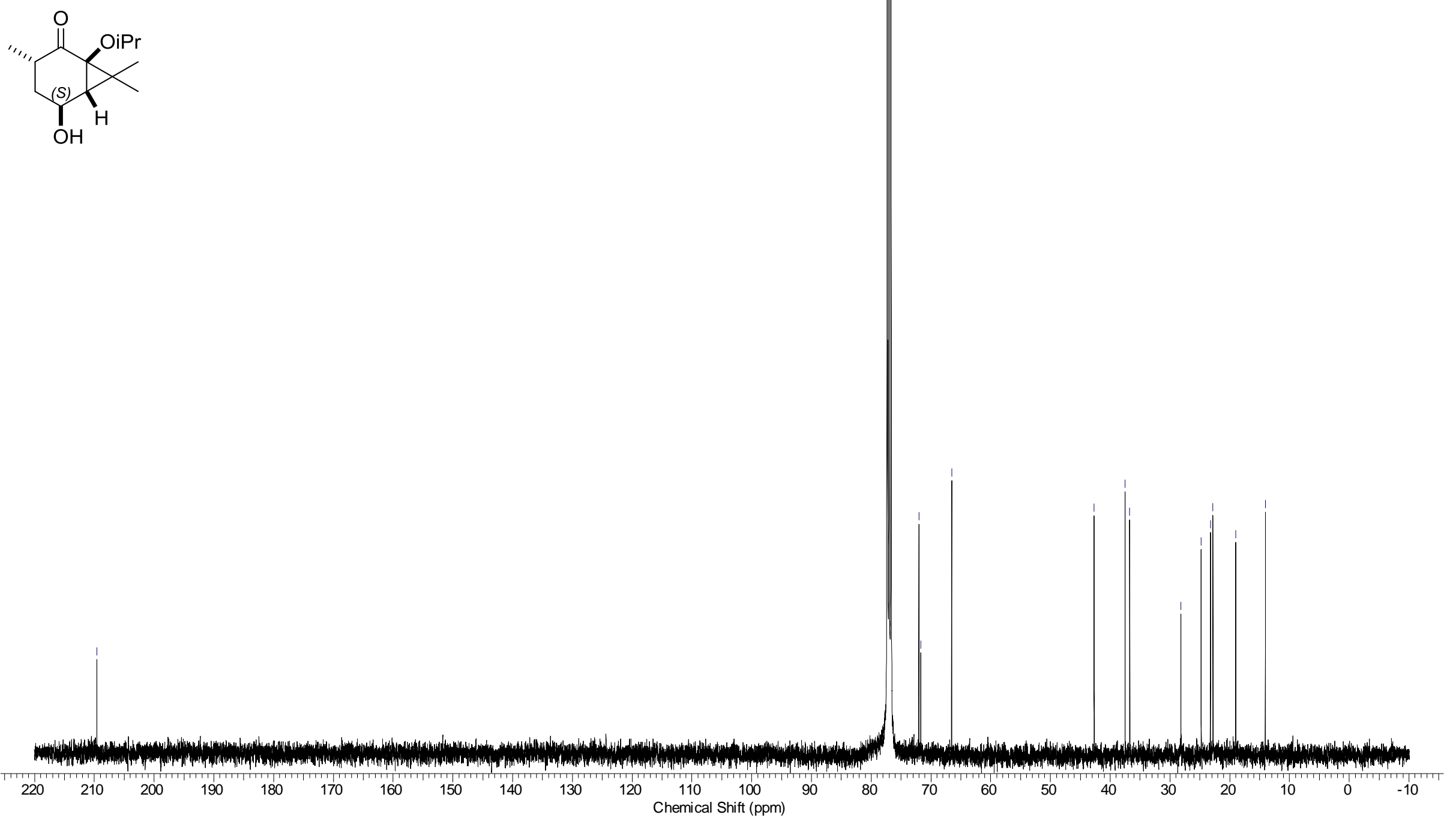

$100 \mathrm{MHz}{ }^{13} \mathrm{C}$ NMR spectrum of $\left(5 S^{*}\right)-22\left(\mathrm{CDCl}_{3}\right)$. 


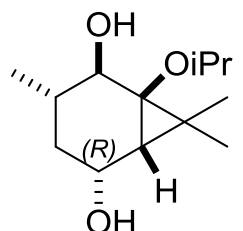

$\mathrm{OH}$

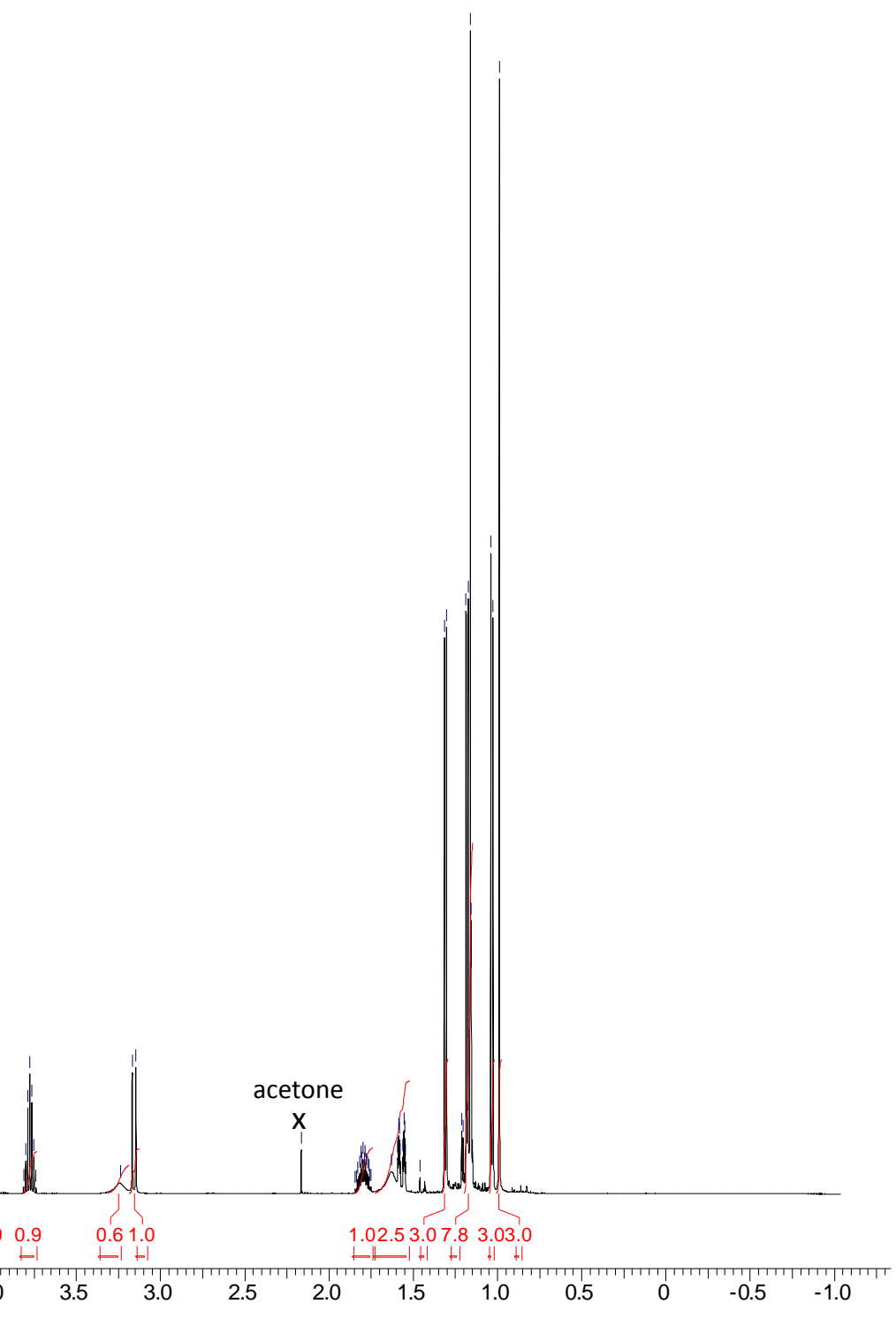

$500 \mathrm{MHz}{ }^{1} \mathrm{H}$ NMR spectrum of $\left(5 R^{*}\right)-23\left(\mathrm{CDCl}_{3}\right)$. 


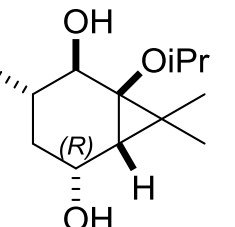

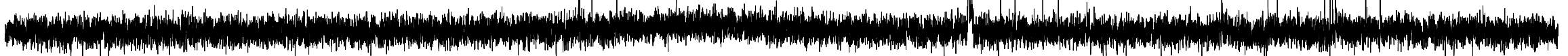

$100 \mathrm{MHz}{ }^{13} \mathrm{C}$ NMR spectrum of $\left(5 R^{*}\right)-23\left(\mathrm{CDCl}_{3}\right)$. 
(")

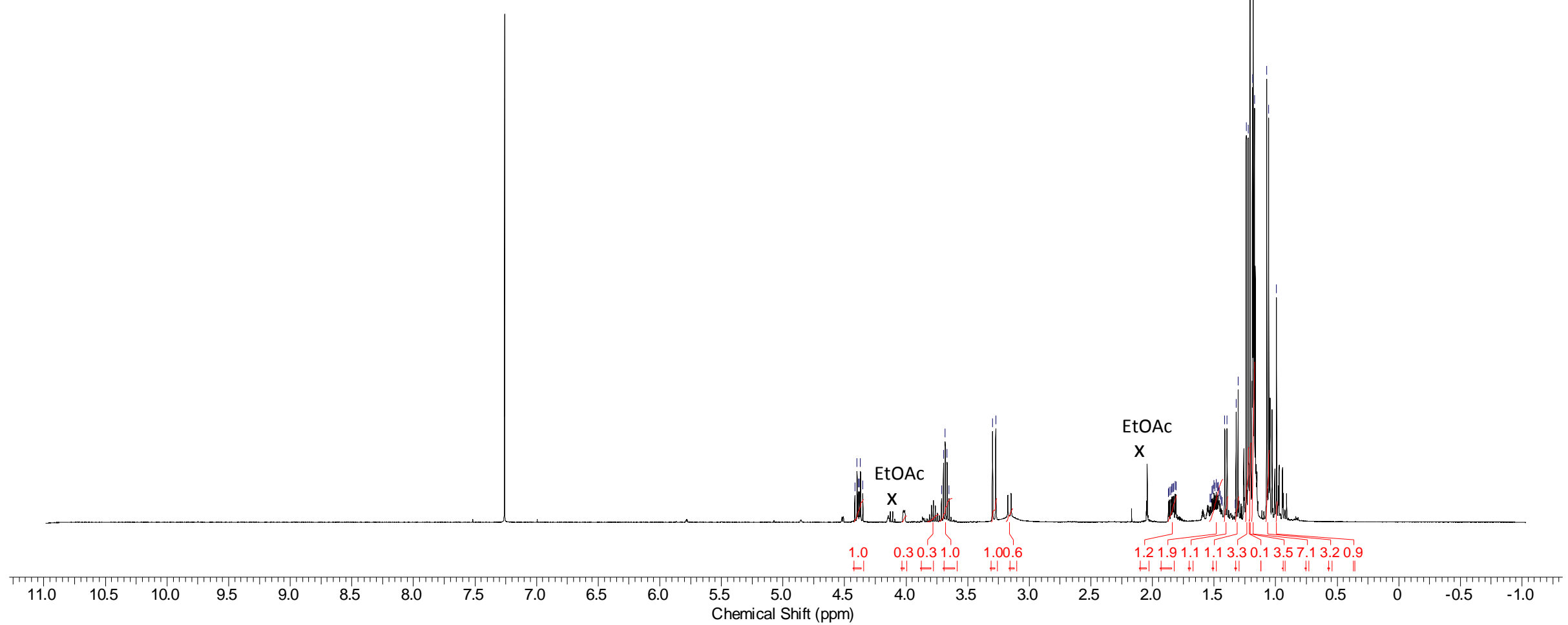

$400 \mathrm{MHz}{ }^{1} \mathrm{H}$ NMR spectrum of $\left(5 S^{*}\right)-23\left(\mathrm{CDCl}_{3}\right)$, containing 23\% of $\left(5 R^{*}\right)-23$. 


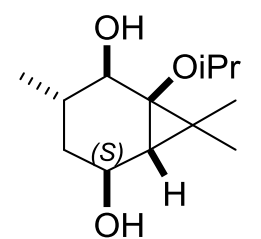

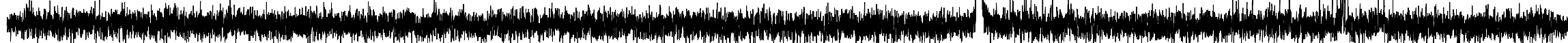
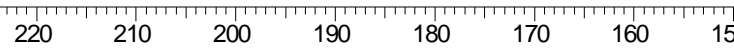

140

$120 \begin{array}{cc}110 & 100 \\ \text { Chemical Shift (ppm) }\end{array}$

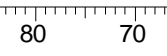

60

40
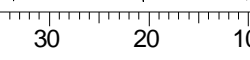

$100 \mathrm{MHz}{ }^{13} \mathrm{C}$ NMR spectrum of $\left(5 S^{*}\right)-23\left(\mathrm{CDCl}_{3}\right)$, containing 23\% of $\left(5 R^{*}\right)-23$. 


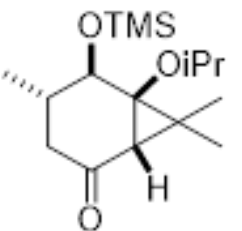

scE026w. 001.001.1. 1.es

O
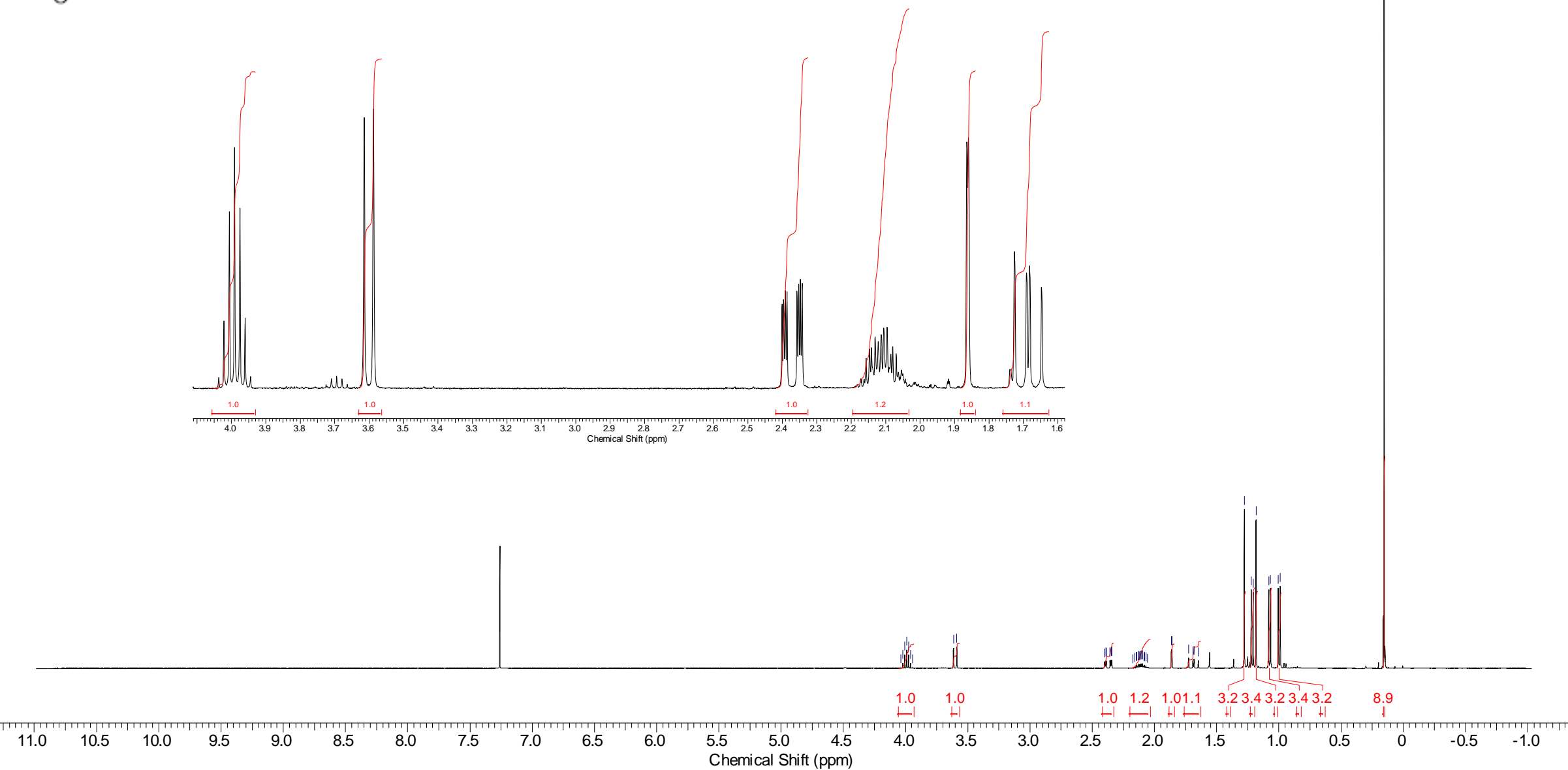

$400 \mathrm{MHz}{ }^{1} \mathrm{H}$ NMR spectrum of $24\left(\mathrm{CDCl}_{3}\right)$. 


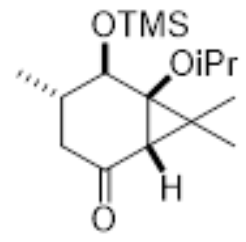

TMS

$\int_{\mathrm{H}}^{\mathrm{OiPr}}$

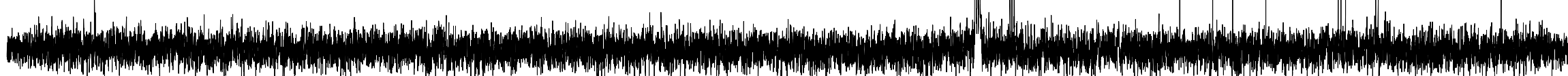

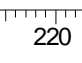

200

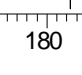

170

150

140

$120 \begin{array}{cc}110 & 100 \\ \text { Chemical Shift (ppm) }\end{array}$

$100 \mathrm{MHz}{ }^{13} \mathrm{C}$ NMR spectrum of $24\left(\mathrm{CDCl}_{3}\right)$. 

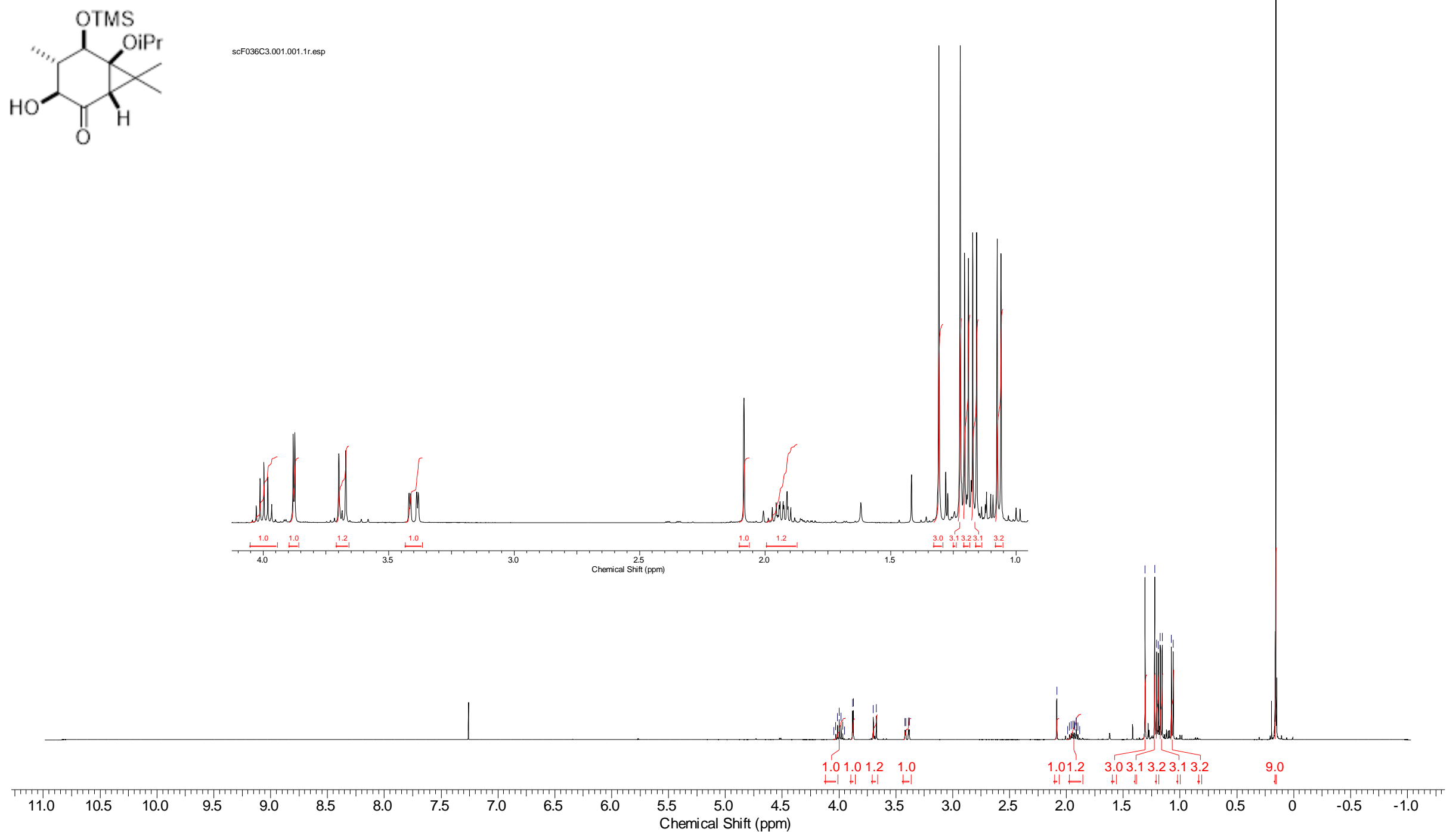

$400 \mathrm{MHz}{ }^{1} \mathrm{H}$ NMR spectrum of $26\left(\mathrm{CDCl}_{3}\right)$. 


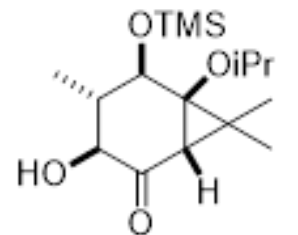

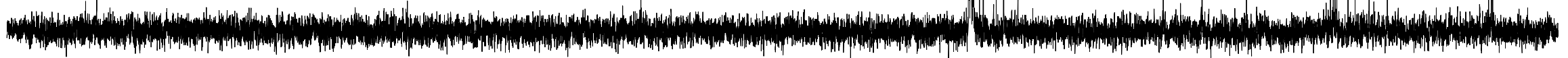

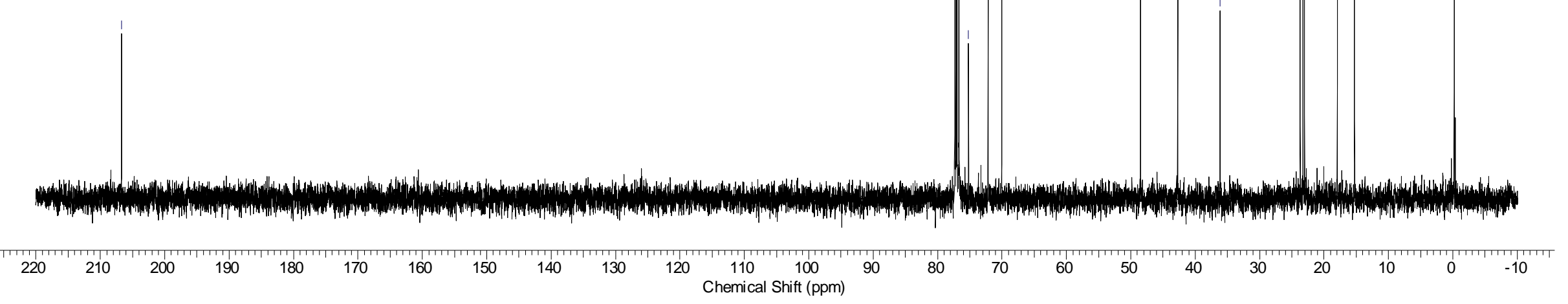

$100 \mathrm{MHz}{ }^{13} \mathrm{C} \mathrm{NMR}$ spectrum of $26\left(\mathrm{CDCl}_{3}\right)$. 

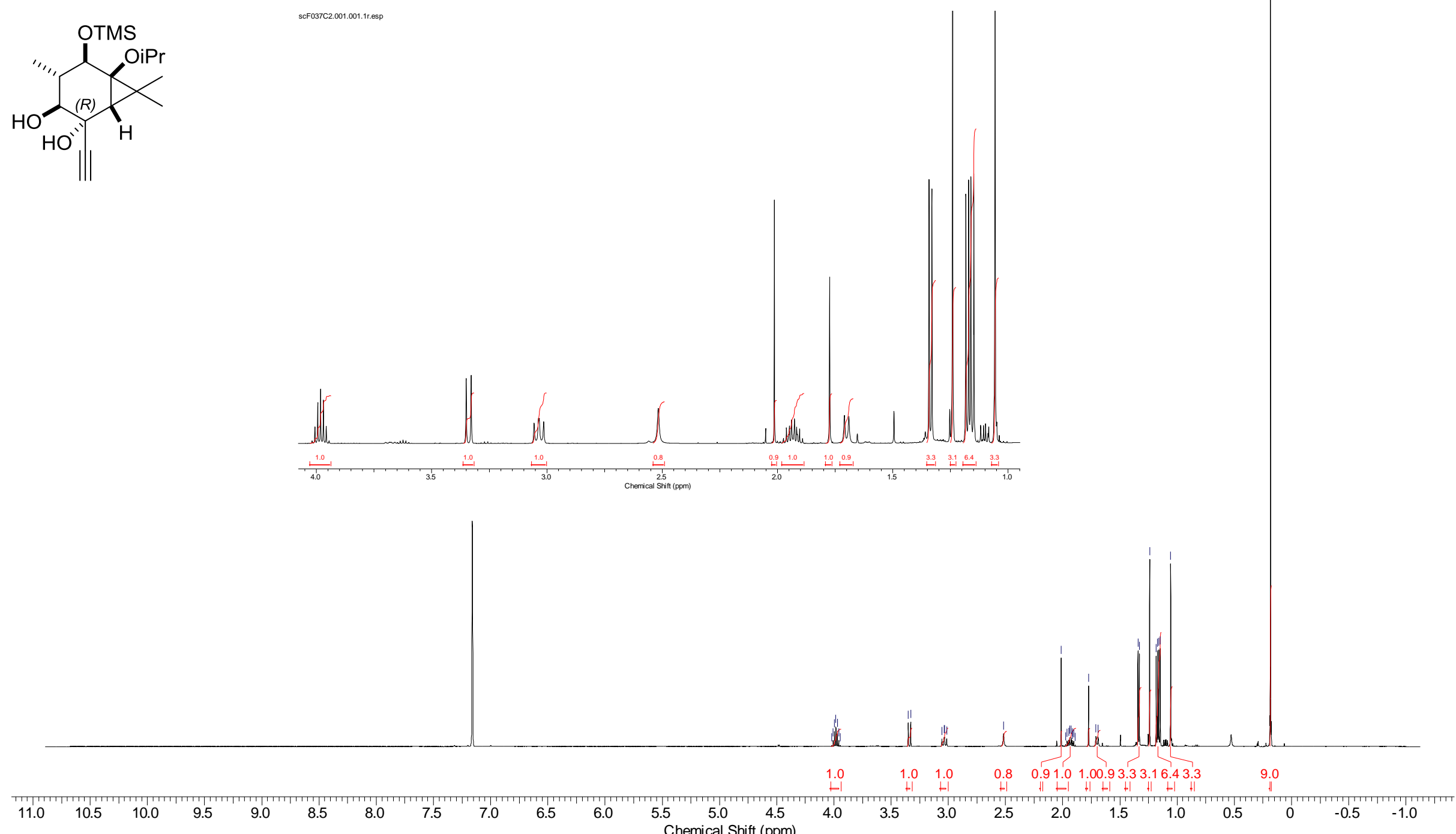

$500 \mathrm{MHz}{ }^{1} \mathrm{H}$ NMR spectrum of $27\left(\mathrm{C}_{6} \mathrm{D}_{6}\right)$. 


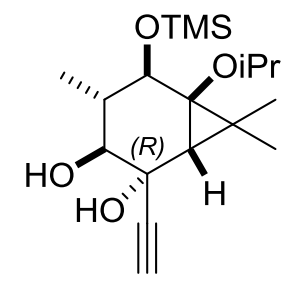

$100 \mathrm{MHz}{ }^{13} \mathrm{C}$ NMR spectrum of $27\left(\mathrm{C}_{6} \mathrm{D}_{6}\right)$. 

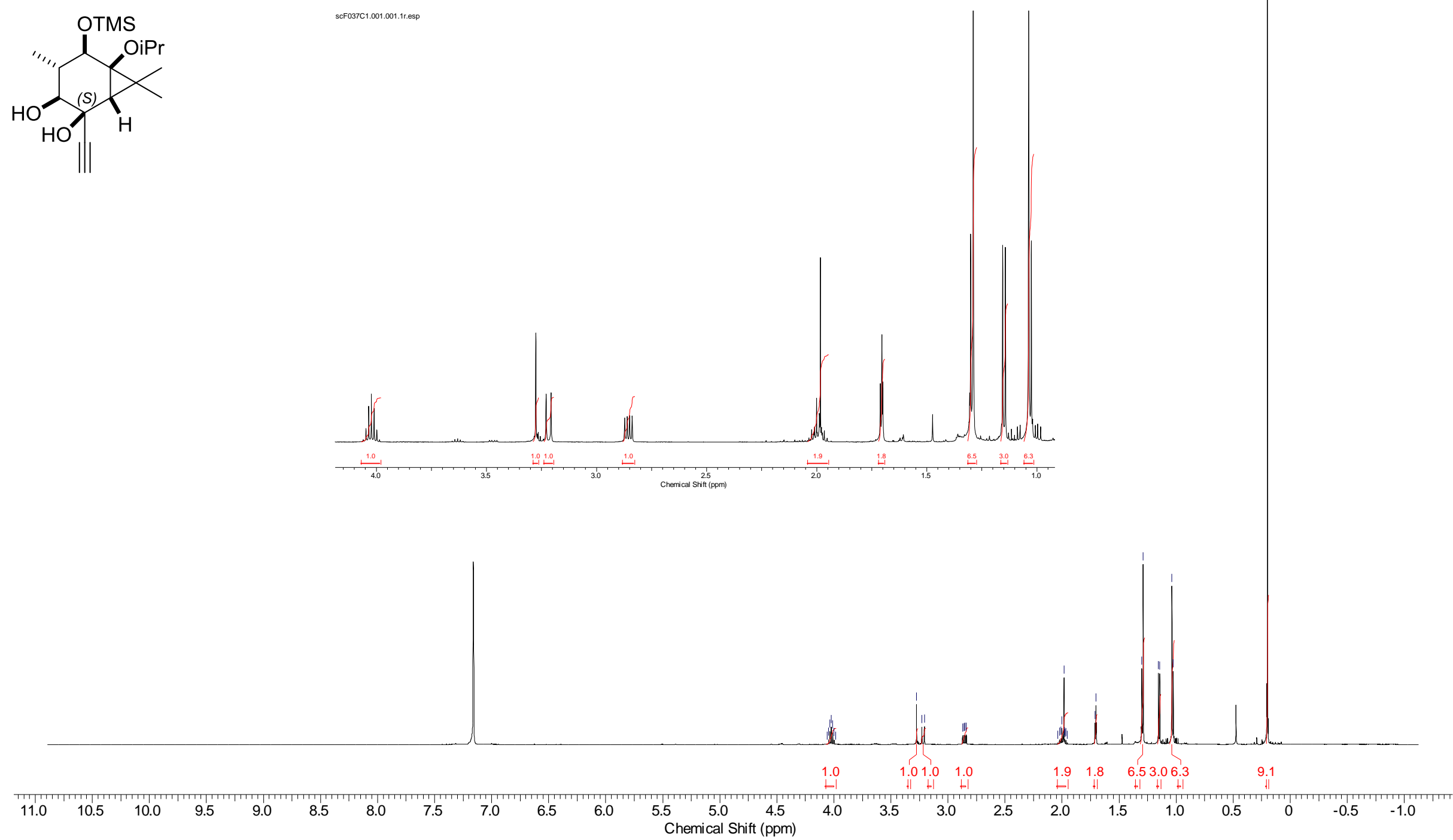

$500 \mathrm{MHz}{ }^{1} \mathrm{H}$ NMR spectrum of $28\left(\mathrm{C}_{6} \mathrm{D}_{6}\right)$. 

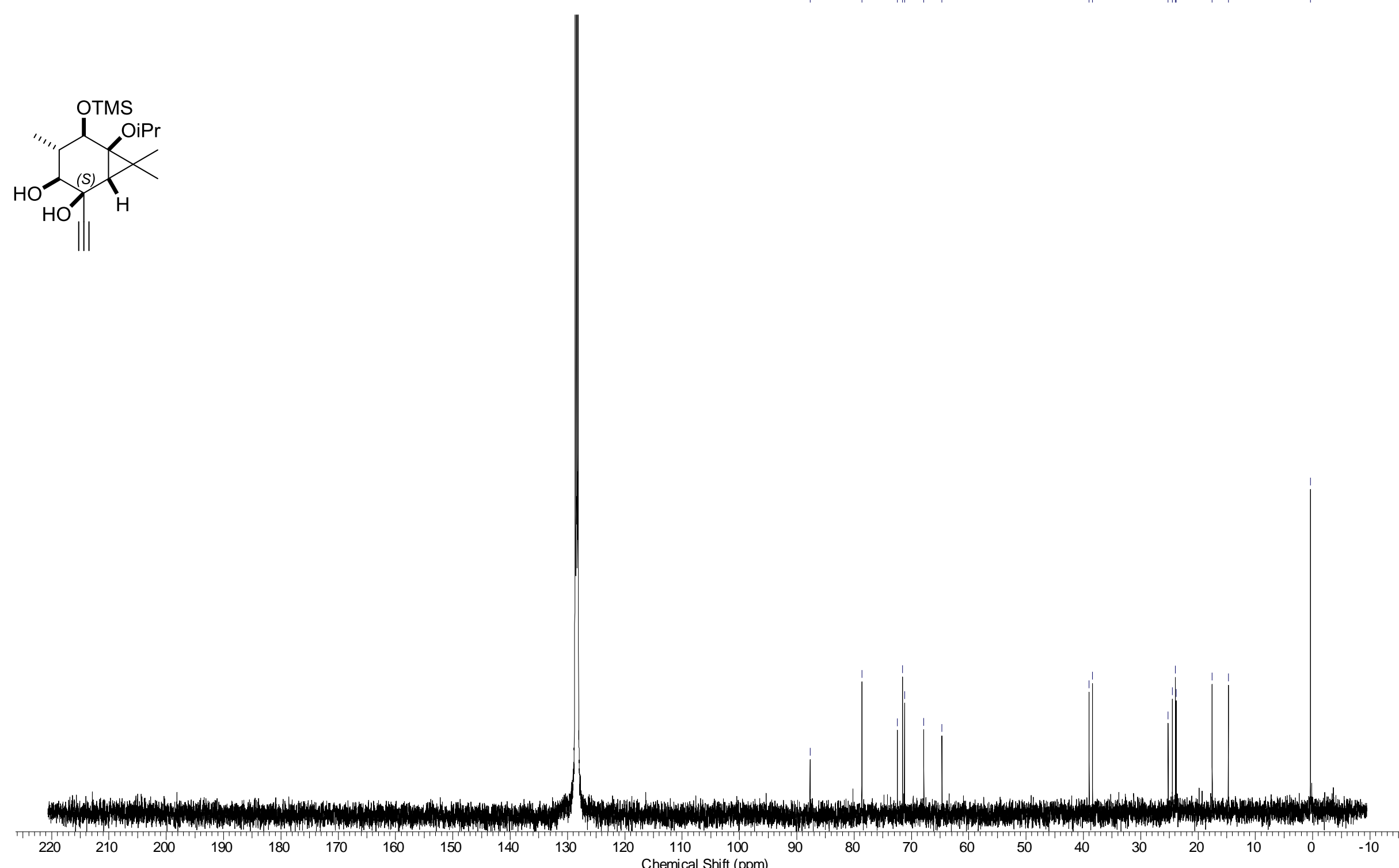

$100 \mathrm{MHz}{ }^{13} \mathrm{C}$ NMR spectrum of $28\left(\mathrm{C}_{6} \mathrm{D}_{6}\right)$. 

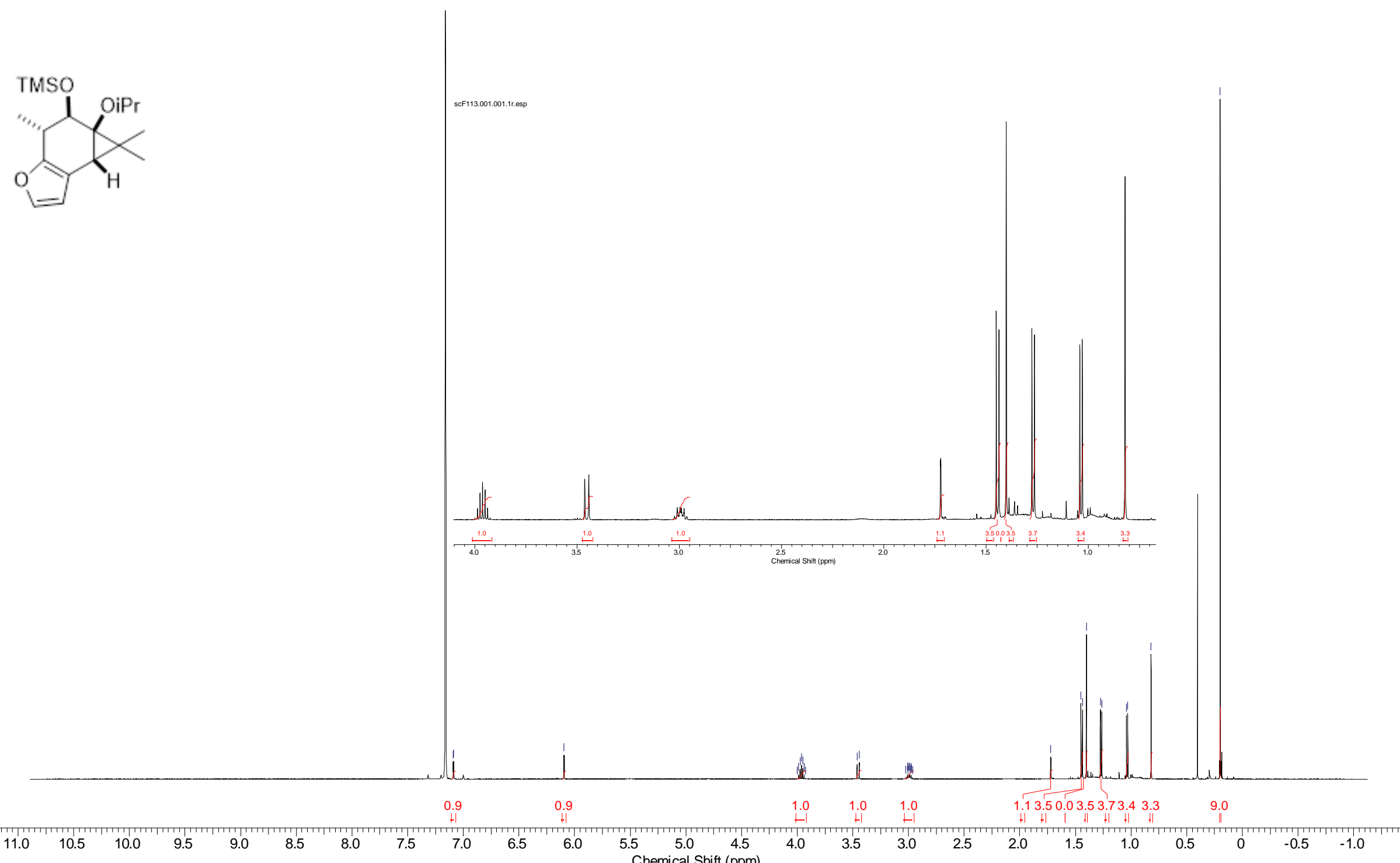

$500 \mathrm{MHz}{ }^{1} \mathrm{H}$ NMR spectrum of $29\left(\mathrm{C}_{6} \mathrm{D}_{6}\right)$. 


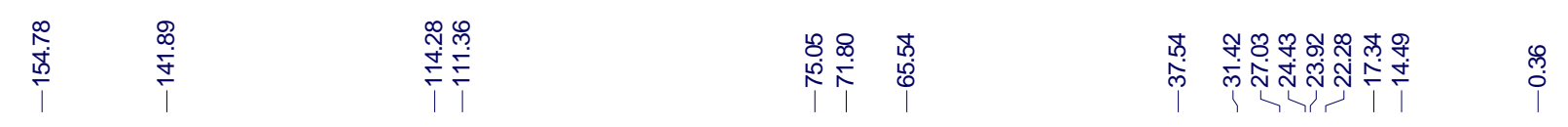

TMSO

$\overbrace{H}^{\text {OiPr }}$

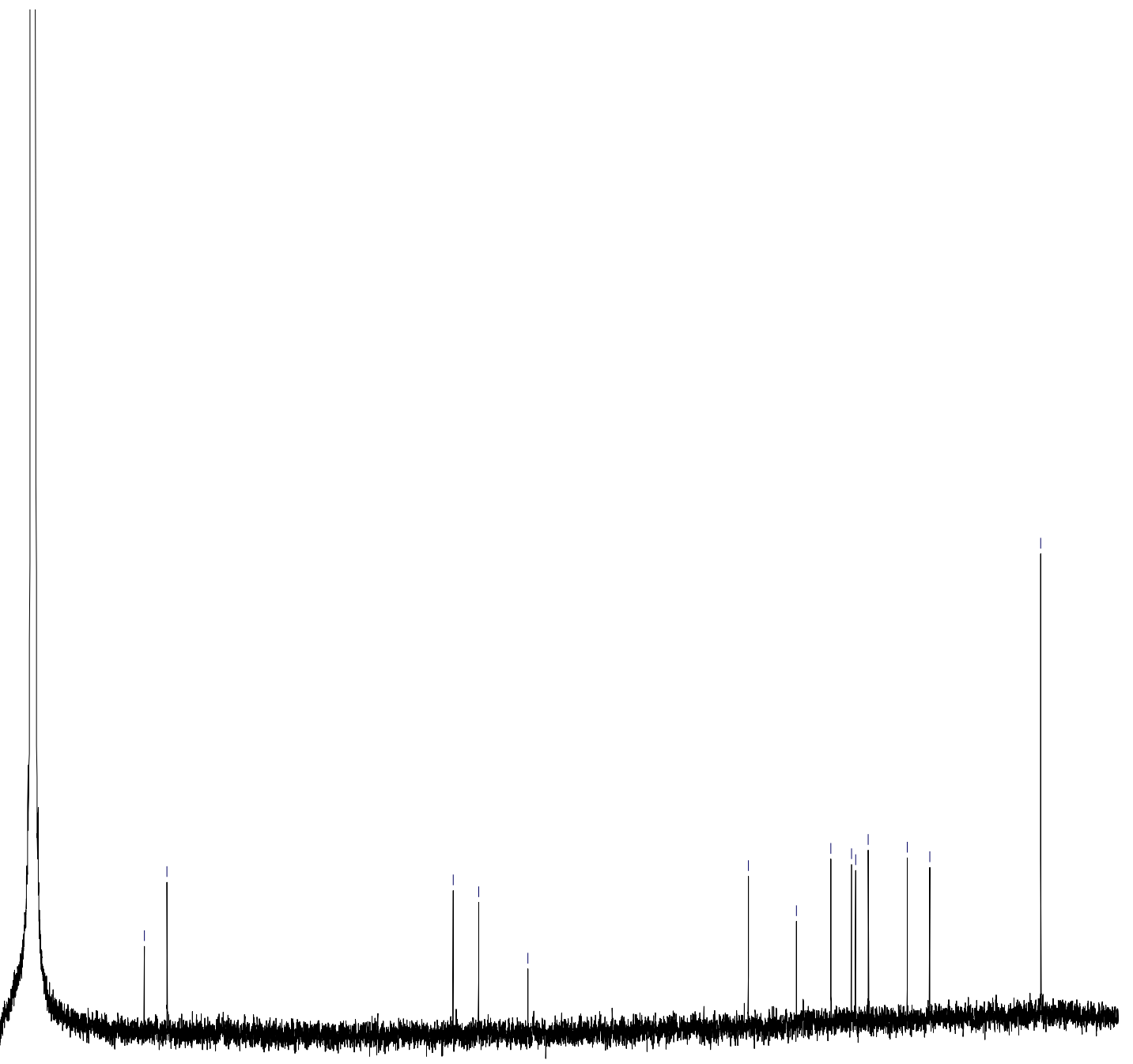

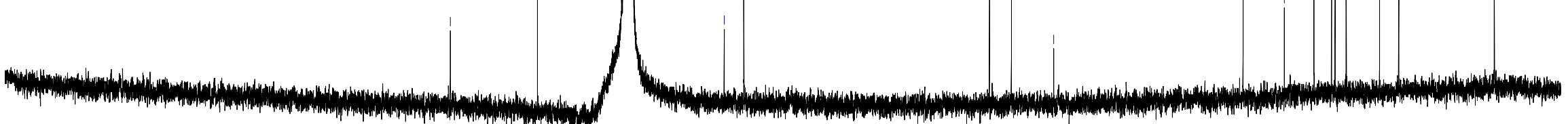

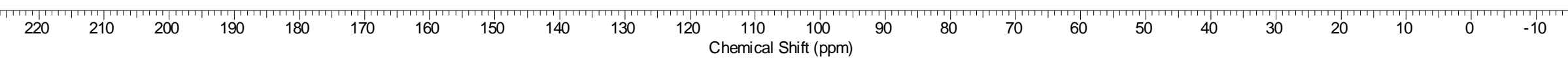

$100 \mathrm{MHz}{ }^{13} \mathrm{C}$ NMR spectrum of $29\left(\mathrm{C}_{6} \mathrm{D}_{6}\right)$. 

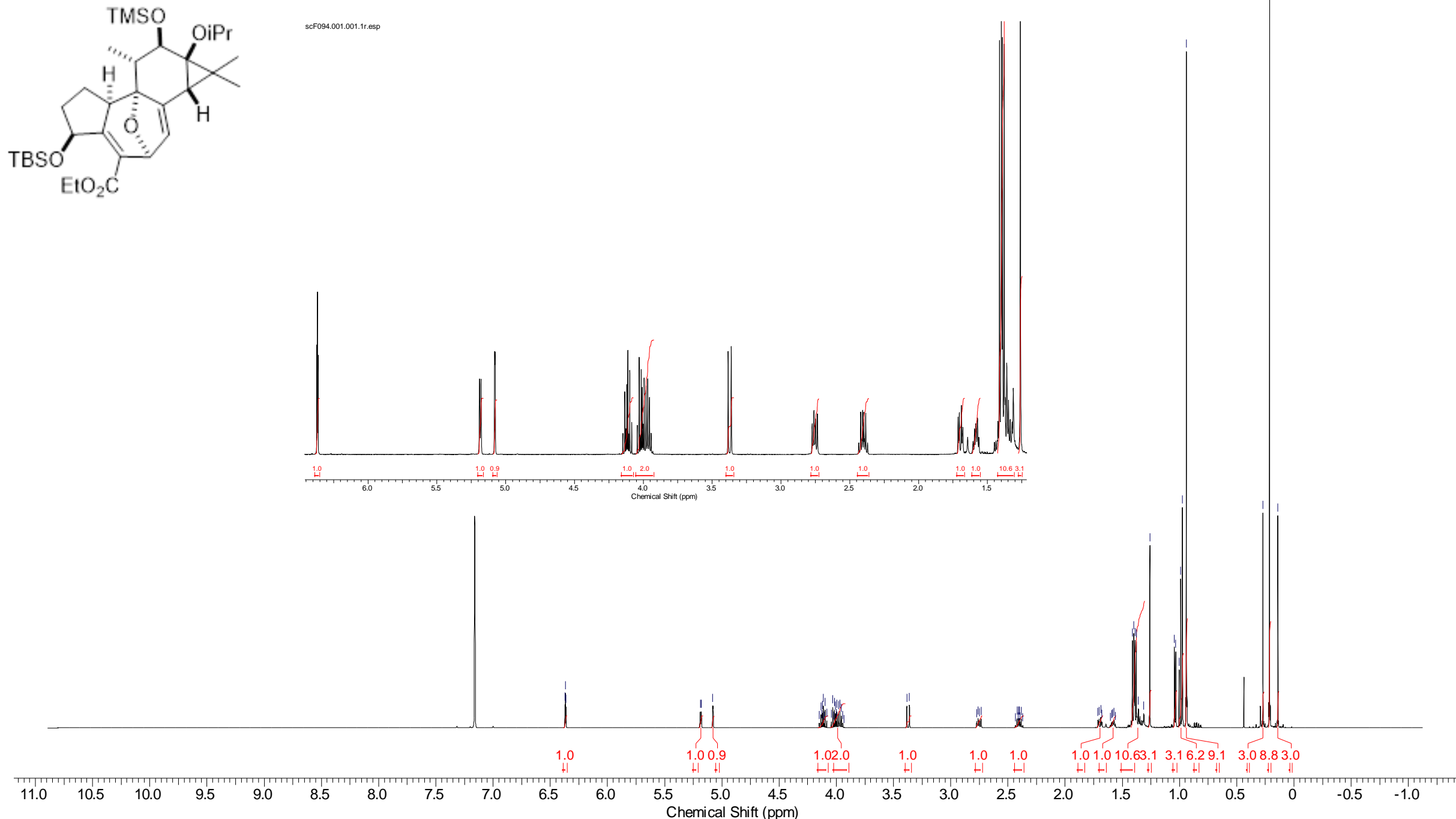

$500 \mathrm{MHz}{ }^{1} \mathrm{H}$ NMR spectrum of $30\left(\mathrm{C}_{6} \mathrm{D}_{6}\right)$ 


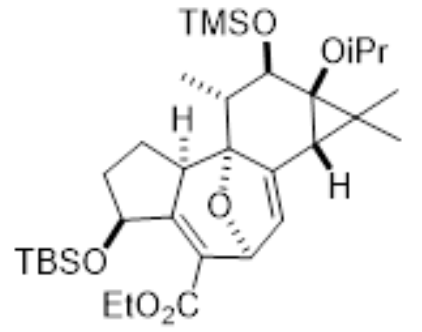

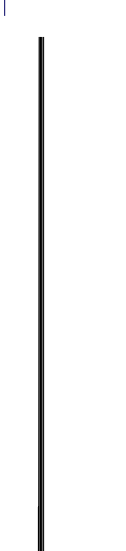

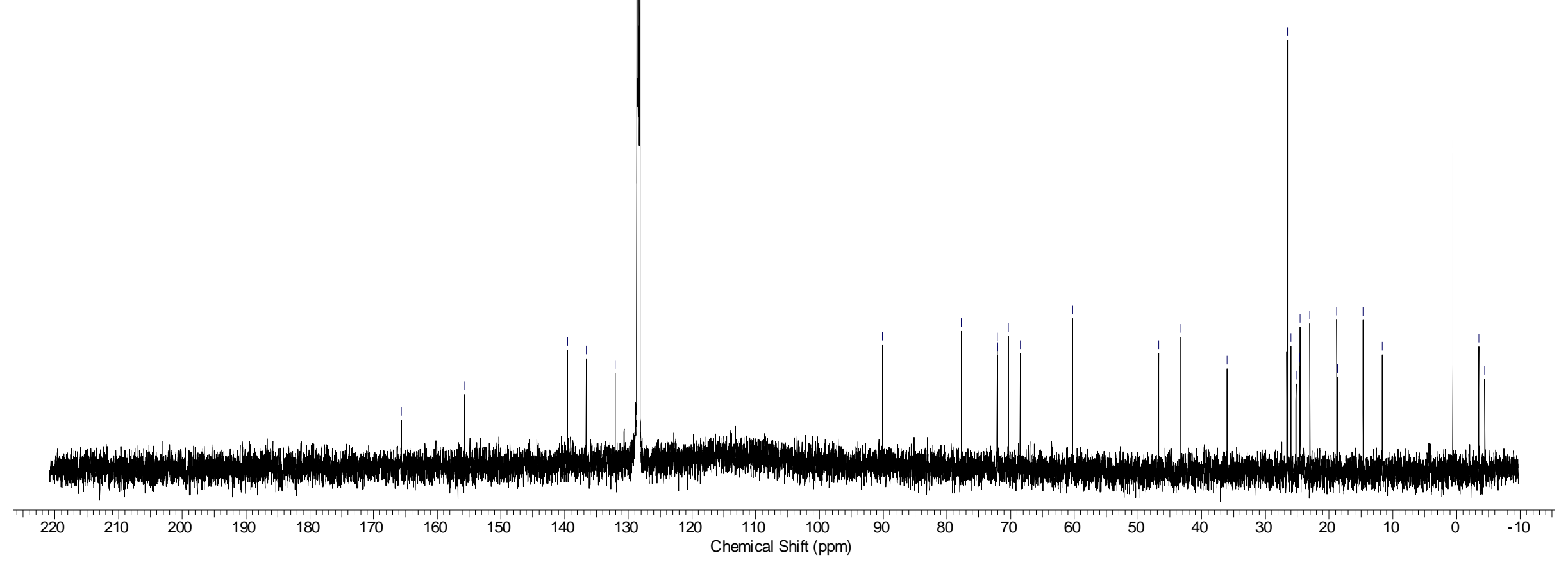

$100 \mathrm{MHz}{ }^{13} \mathrm{C}$ NMR spectrum of $30\left(\mathrm{C}_{6} \mathrm{D}_{6}\right)$. 

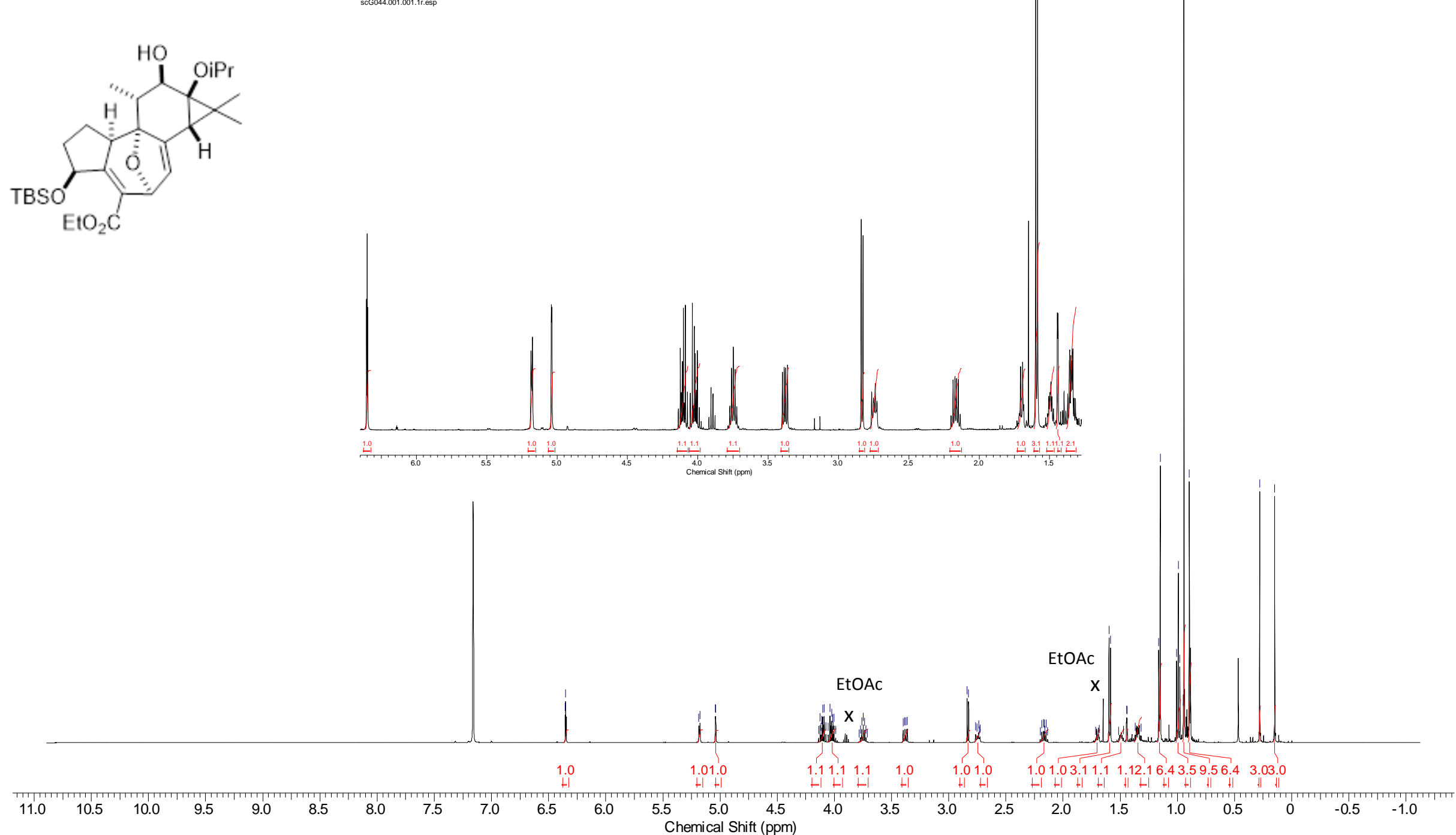

$500 \mathrm{MHz}{ }^{1} \mathrm{H}$ NMR spectrum of $31\left(\mathrm{C}_{6} \mathrm{D}_{6}\right)$. 


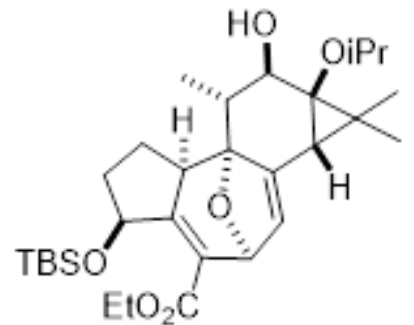

$$
\mid
$$




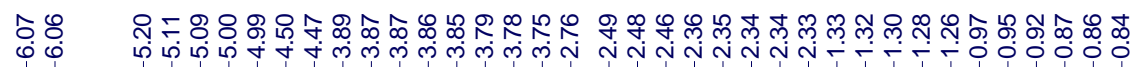
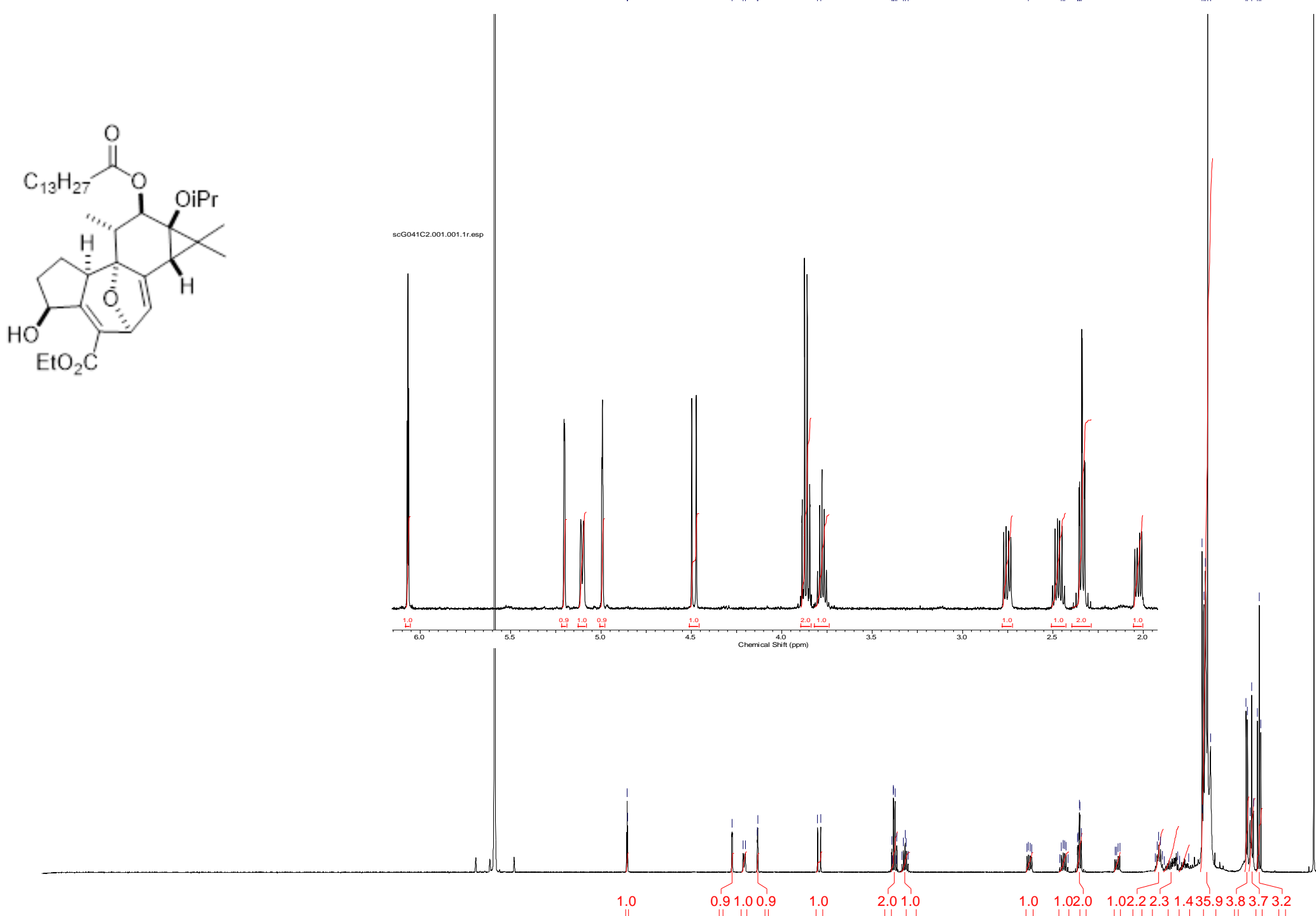

$\begin{array}{lll}1.0 & 1.02 .0 & 1.02 .22 .31 .435 .93 .83 .73 .2\end{array}$

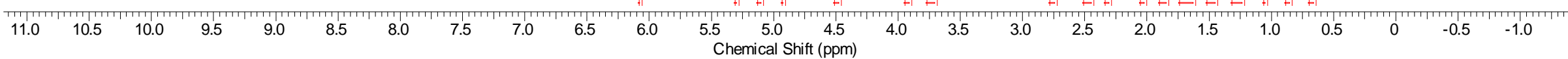

$500 \mathrm{MHz}{ }^{1} \mathrm{H}$ NMR spectrum of $32\left(\mathrm{C}_{6} \mathrm{D}_{6}\right)$. 

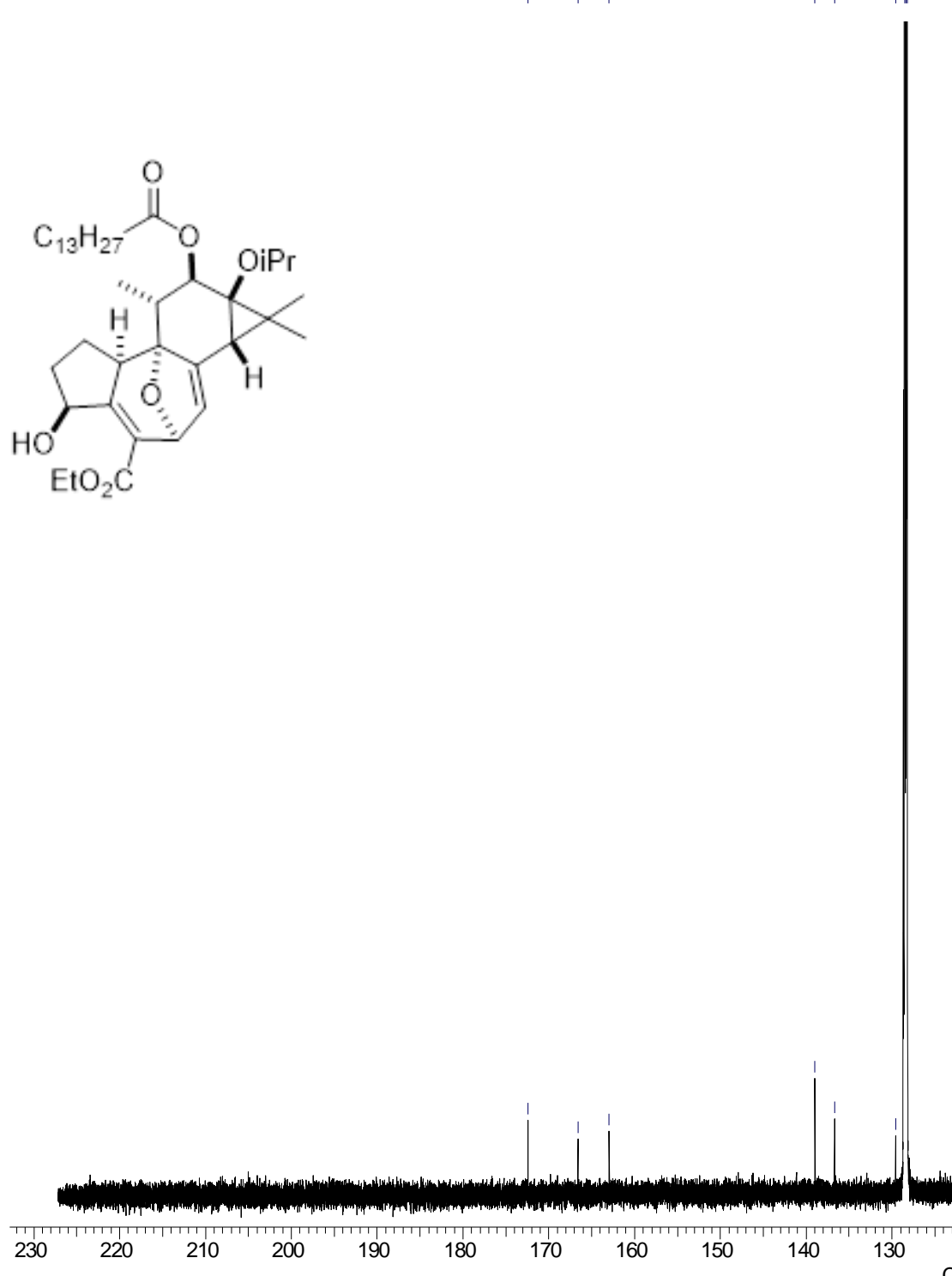

$160 \quad 150$
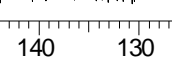

$\begin{array}{llll}120 & 10 & 100 \\ \text { Chemical Shift (ppm) }\end{array}$

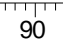

80

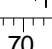

$70 \quad 60$

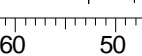

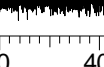

$40 \quad 30$

$176 \mathrm{MHz}{ }^{13} \mathrm{C}$ NMR spectrum of $32\left(\mathrm{C}_{6} \mathrm{D}_{6}\right)$. 

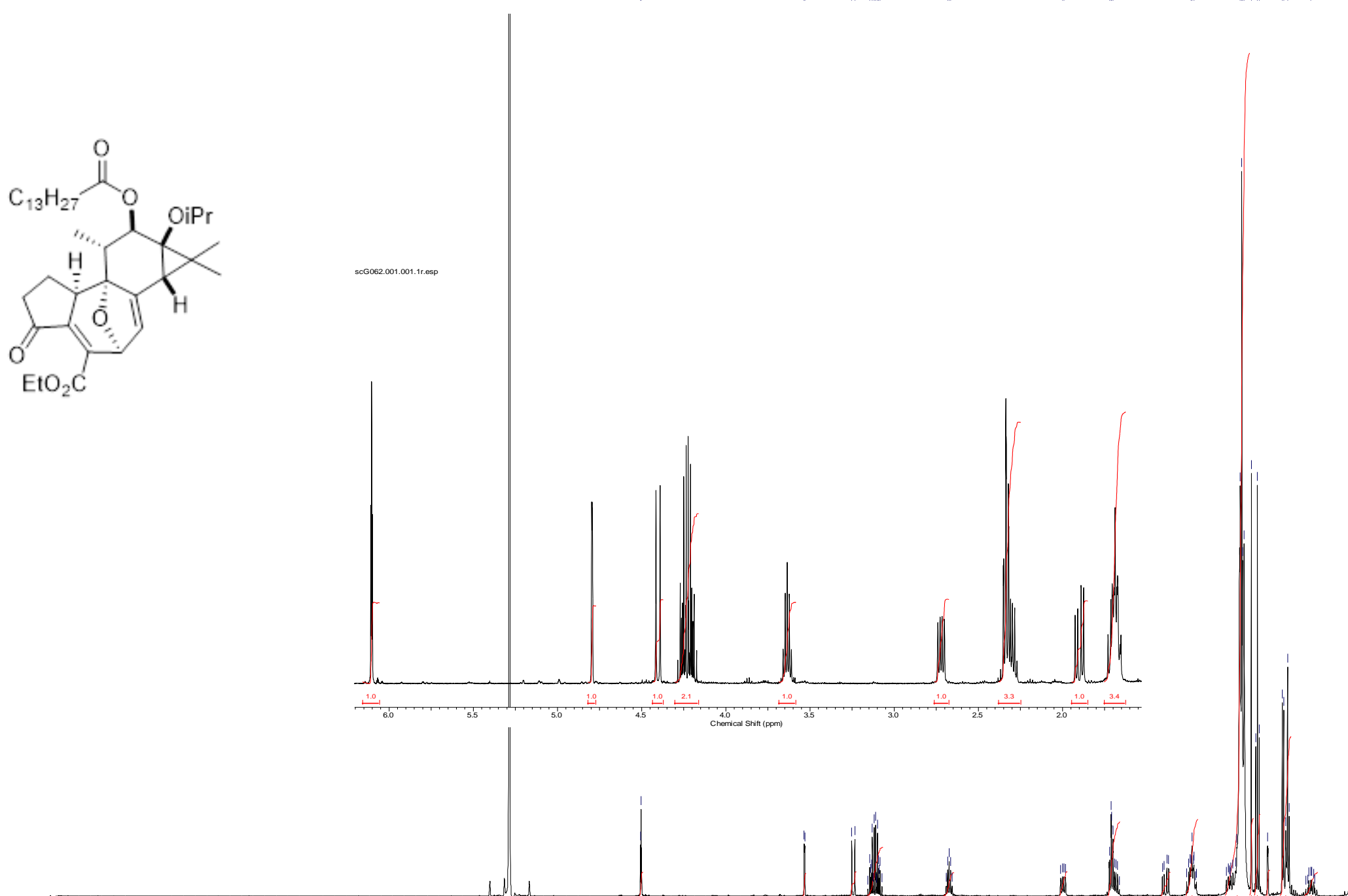

1.0

$0.9 \quad 1.02$. $\begin{array}{lll}.0 & 3.2 & 1.03 .336 .33 .33 .51 .16 .81 .0\end{array}$ 


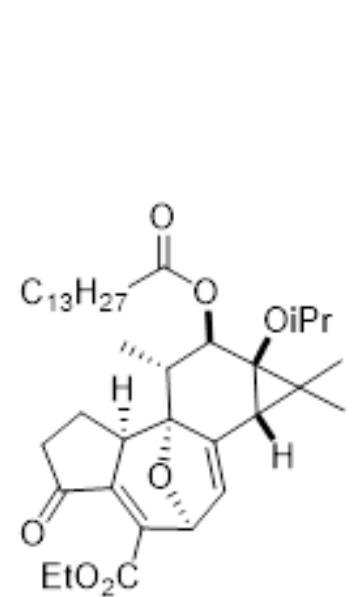

$\mathrm{EtO}_{2} \mathrm{C}$
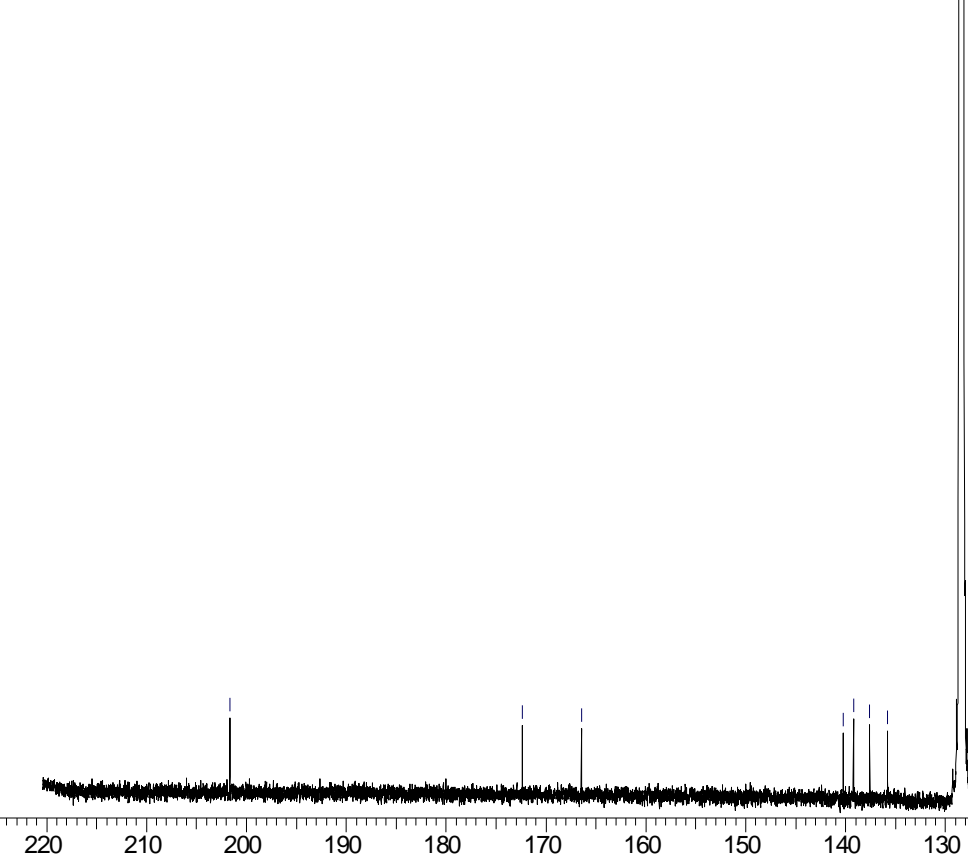

$125 \mathrm{MHz}{ }^{13} \mathrm{C}$ NMR spectrum of $33\left(\mathrm{C}_{6} \mathrm{D}_{6}\right)$. 